\title{
Three-Body Scattering at Intermediate Energies
}

\author{
H. Liu, Ch. Elster \\ Institute of Nuclear and Particle Physics, \\ and Department of Physics and Astronomy, \\ Ohio University, Athens, $\mathrm{OH} 45701$ \\ W. Glöckle \\ Institute for Theoretical Physics II, Ruhr-University Bochum, D-44780 Bochum, Germany.
}

(Dated: November 6, 2018)

\begin{abstract}
The Faddeev equation for three-body scattering at arbitrary energies is formulated in momentum space and directly solved in terms of momentum vectors without employing a partial wave decomposition. In its simplest form the Faddeev equation for identical bosons, which we are using, is a three-dimensional integral equation in five variables, magnitudes of relative momenta and angles. This equation is solved through Padé summation. Based on a Malfliet-Tjon-type potential, the numerical feasibility and stability of the algorithm for solving the Faddeev equation is demonstrated. Special attention is given to the selection of independent variables and the treatment of three-body break-up singularities with a spline based method. The elastic differential cross section, semi-exclusive d $\left(\mathrm{N}, \mathrm{N}^{\prime}\right)$ cross sections and total cross sections of both elastic and breakup processes in the intermediate energy range up to about $1 \mathrm{GeV}$ are calculated and the convergence of the multiple scattering series is investigated in every case. In general a truncation in the first or second order in the two-body t-matrix is quite insufficient.
\end{abstract}

PACS numbers: $21.45+\mathrm{v}$

\section{INTRODUCTION}

During the last two decades calculations of nucleon-deuteron scattering experienced large improvements and refinements. Here different techniques have been applied, Faddeev calculations in configuration space [1] and momentum space [2], and variational calculations based on a hyperspherical harmonic expansion [3, 4]. It is fair to say that below about $200 \mathrm{MeV}$ projectile energy the momentum space Faddeev equations for three-nucleon scattering can now be solved with high accuracy for the most modern two- and three-nucleon forces. A summary for these achievements can be found in Ref. [5, 6, 7, 8, 9]. The approach described there is based on using angular momentum eigenstates for the two- and three-body systems. This partial wave decomposition replaces the continuous angle variables by discrete orbital angular momentum quantum numbers, and thus reduces the number of continuous variables to be discretized in a numerical treatment. For low projectile energies the procedure of considering orbital angular momentum components appears physically justified due to arguments related to the centrifugal barrier and the short range of the nuclear force. However, the algebraic and algorithmic steps to be carried out in a partial wave decomposition can be quite involved when solving the Faddeev equations. If one considers three-nucleon scattering at a few hundred $\mathrm{MeV}$ projectile energy, the number of partial waves needed to achieve convergence proliferates, and limitations with respect to computational feasibility and accuracy are reached.

It appears therefore natural to avoid a partial wave representation completely and work directly with vector variables. This is common practice in bound state calculations of few-nucleon systems based on variational [10] and Green's function Monte Carlo (GFMC) methods [11, 12, 13, 14], which are carried out in configuration space.

Our aim is to work directly with vector variables in the Faddeev scheme in momentum space. In earlier work 15, 16 we showed that the bound state Faddeev equation has a rather transparent structure when formulated with vector variables compared to the coupled set of two-dimensional integral equations obtained in a partial wave decomposed form. Based on Malfliet-Tjon type interactions for two- as well as three-body forces, it was demonstrated that the numerical solution of the bound state equation using vector variables is straightforward and numerically very accurate. As far as three nucleon scattering is concerned, the neutron-deuteron break-up process has been successfully studied up to $500 \mathrm{MeV}$ projectile energy based on the first order term of the Faddeev equation using realistic nucleon-nucleon forces 17].

In this article we want to show that the full solution of the three-body scattering equation can be obtained in a straightforward manner when employing vector variables, i.e. magnitudes of momenta and angles between the momentum vectors. As a simplification we neglect spin and iso-spin degrees of freedom and treat three-boson scattering. The interactions employed are of Yukawa type, and no separable approximations are involved. The Faddeev equation for three identical bosons is solved exactly as function of momentum vectors below and above the three-body break-up.

This article is organized as follows. Section II reviews the Faddeev equation for three-body scattering in momentum 
space and discusses our choice of momentum and angle variables for the unknown amplitude in the equation and its kernel. In Section III we derive the amplitudes and cross sections for elastic scattering and break-up processes. In addition we relate both via the optical theorem. In Section IV we discuss the numerical methods necessary for solving the Faddeev equation, especially our treatment of the singularities in the free three-body propagator. In addition, our numerical tests for the solution are shown and discussed. In Section $\mathrm{V}$ we present calculations for elastic scattering and break-up processes in the intermediate energy regime from 0.2 to $1 \mathrm{GeV}$. Our focus here is on the study of the importance of rescattering terms as function of the projectile energy and the reaction considered. We conclude in Section VI.

\section{FADDEEV EQUATIONS FOR THREE BOSONS IN THE CONTINUUM}

Various presentations of three-body scattering in the Faddeev scheme are presented in the literature [5, 6, 18]. We solve the Faddeev equation for three identical particles in the form

$$
T|\phi\rangle=t P|\phi\rangle+t P G_{0} T|\phi\rangle
$$

The driving term of this integral equation consists of the two-body t-matrix $t$, the sum $P$ of a cyclic and anticyclic permutation of three particles, and the initial state $|\phi\rangle=\left|\varphi_{d} \mathbf{q}_{\mathbf{0}}\right\rangle$, composed of a two-body bound state and the momentum eigenstate of the projectile particle. The kernel of of Eq. 2.1] contains the free three-body propagator, $G_{0}=\left(E-H_{0}+i \varepsilon\right)^{-1}$, where $E$ is the total energy in the center of mass frame.

The operator $T$ determines both, the full break-up amplitude

$$
U_{0}=(1+P) T
$$

and the amplitude for elastic scattering

$$
U=P G_{0}^{-1}+P T
$$

In this paper we focus on three identical bosons and use a momentum space representation. For solving Eq. (2.1), we introduce standard Jacobi momenta $\mathbf{p}$, the relative momentum in the subsystem, and $\mathbf{q}$, the relative momentum of the spectator to the subsystem. The momentum states are normalized according to $\left\langle\mathbf{p}^{\prime} \mathbf{q}^{\prime} \mid \mathbf{p q}\right\rangle=\delta^{3}\left(\mathbf{p}^{\prime}-\mathbf{p}\right) \delta^{3}\left(\mathbf{q}^{\prime}-\mathbf{q}\right)$. Projecting Eq. (2.1) on to Jacobi momenta leads to [19]

$$
\begin{aligned}
\left\langle\mathbf{p q}|T| \varphi_{d} \mathbf{q}_{0}\right\rangle & =\varphi_{d}\left(\mathbf{q}+\frac{1}{2} \mathbf{q}_{0}\right) t_{s}\left(\mathbf{p}, \frac{1}{2} \mathbf{q}+\mathbf{q}_{0}, E-\frac{3}{4 m} q^{2}\right) \\
& +\int d^{3} q^{\prime \prime} t_{s}\left(\mathbf{p}, \frac{1}{2} \mathbf{q}+\mathbf{q}^{\prime \prime}, E-\frac{3}{4 m} q^{2}\right) \frac{\left\langle\mathbf{q}+\frac{1}{2} \mathbf{q}^{\prime \prime}, \mathbf{q}^{\prime \prime}|T| \varphi_{d} \mathbf{q}_{0}\right\rangle}{E-\frac{1}{m}\left(q^{2}+q^{\prime \prime 2}+\mathbf{q} \cdot \mathbf{q}^{\prime \prime}\right)+i \varepsilon}
\end{aligned}
$$

Here $t_{s}\left(\mathbf{p}^{\prime}, \mathbf{p}\right)=t\left(\mathbf{p}, \mathbf{p}^{\prime}\right)+t\left(-\mathbf{p}^{\prime}, \mathbf{p}\right)$ is the symmetrized $t$ matrix and $E$ is the total energy in the center of mass (c.m.) system

$$
E=E_{d}+\frac{3}{4 m} q_{0}^{2}=E_{d}+\frac{2}{3} E_{l a b} .
$$

We assume that the underlying force is a two-body force, generating $t$ via a two-body Lippmann-Schwinger equation and supporting one bound state with energy $E_{d}$. Thus, $t_{s}(z)$ has a pole at $z=E_{d}$. Since the transition operator $T$ in Eq. (2.4) is needed for all values of $\mathbf{q}$, one encounters this pole of $t_{s}$. Extracting the residue explicitly by defining

$$
t_{s}\left(\mathbf{p}^{\prime}, \mathbf{p}, z\right) \equiv \frac{\hat{t}_{s}\left(\mathbf{p}^{\prime}, \mathbf{p}, z\right)}{z-E_{d}}
$$

and similarly for T, Eq. (2.4) can be rewritten as

$$
\begin{aligned}
\left\langle\mathbf{p q}|\hat{T}| \varphi_{d} \mathbf{q}_{0}\right\rangle & =\varphi_{d}\left(\mathbf{q}+\frac{1}{2} \mathbf{q}_{0}\right) \hat{t}_{s}\left(\mathbf{p}, \frac{1}{2} \mathbf{q}+\mathbf{q}_{0}, E-\frac{3}{4 m} q^{2}\right) \\
& +\int d^{3} q^{\prime \prime} \frac{\hat{t}_{s}\left(\mathbf{p}, \frac{1}{2} \mathbf{q}+\mathbf{q}^{\prime \prime}, E-\frac{3}{4 m} q^{2}\right)}{E-\frac{1}{m}\left(q^{2}+q^{\prime \prime 2}+\mathbf{q} \cdot \mathbf{q}^{\prime \prime}\right)+i \varepsilon} \frac{\left\langle\mathbf{q}+\frac{1}{2} \mathbf{q}^{\prime \prime}, \mathbf{q}^{\prime \prime}|\hat{T}| \varphi_{d} \mathbf{q}_{0}\right\rangle}{E-\frac{3}{4 m} q^{\prime \prime 2}-E_{d}+i \varepsilon}
\end{aligned}
$$


This expression is the starting point for our numerical calculation of the transition amplitude without employing an angular momentum decomposition.

The first important step for an explicit calculation is the selection of independent variables. Since we ignore spin and iso-spin dependencies, the matrix element $\left\langle\mathbf{p q}|\hat{T}| \varphi_{d} \mathbf{q}_{\mathbf{0}}\right\rangle$ is a scalar function of the variables $\mathbf{p}$ and $\mathbf{q}$ for a given projectile momentum $\mathbf{q}_{0}$. Thus one needs 5 variables to uniquely specify the geometry of the three vectors $\mathbf{p}$, $\mathbf{q}$ and $\mathbf{q}_{0}$, which are shown in Fig. 1. Having in mind that with three vectors one can span 2 planes, i.e. the $\mathbf{p}$ - $\mathbf{q}_{0}$-plane and the $\mathbf{q}-\mathbf{q}_{0}$-plane, a natural choice of independent variables is

$$
p=|\mathbf{p}|, q=|\mathbf{q}|, x_{p}=\hat{\mathbf{p}} \cdot \hat{\mathbf{q}}_{0}, x_{q}=\hat{\mathbf{q}} \cdot \hat{\mathbf{q}}_{0}, x_{p q}^{q_{0}}=\left(\widehat{\mathbf{q}_{0} \times \mathbf{q}}\right) \cdot\left(\widehat{\mathbf{q}_{0} \times \mathbf{p}}\right) .
$$

The last variable, $x_{p q}^{q_{0}}$, is the angle between the two normal vectors of the $\mathbf{p}$ - $\mathbf{q}_{0}$-plane and the $\mathbf{q}$ - $\mathbf{q}_{0}$-plane, which are explicitly given by

$$
\begin{aligned}
& \left(\widehat{\mathbf{q}_{0} \times} \mathbf{p}\right)=\frac{\hat{\mathbf{q}}_{0} \times \hat{\mathbf{p}}}{\sqrt{1-\left(\hat{\mathbf{q}}_{0} \cdot \hat{\mathbf{p}}\right)^{2}}} \\
& \left(\widehat{\mathbf{q}_{0} \times \mathbf{q}}\right)=\frac{\hat{\mathbf{q}}_{0} \times \hat{\mathbf{q}}}{\sqrt{1-\left(\hat{\mathbf{q}}_{0} \cdot \hat{\mathbf{q}}\right)^{2}}}
\end{aligned}
$$

It should be pointed out, that the angle between the vectors $\mathbf{q}$ and $\mathbf{p}, y_{p q}=\hat{\mathbf{p}} \cdot \hat{\mathbf{q}}$, is not an independent variable. In fact, if $x_{p}$ and $x_{q}$ are given, the domain of $y_{p q}$ is bound by

$$
x_{p} x_{q}-\sqrt{1-x_{p}^{2}} \sqrt{1-x_{q}^{2}} \leq y_{p q} \leq x_{p} x_{q}+\sqrt{1-x_{p}^{2}} \sqrt{1-x_{q}^{2}}
$$

thus not covering the entire interval $[-1,1]$. Using the explicit representation of the normal vectors and standard cross product identities, we arrive at the following relation between $x_{p q}^{q_{0}}$ and $y_{p q}$,

$$
\begin{aligned}
x_{p q}^{q_{0}} & =\frac{\hat{\mathbf{p}} \cdot \hat{\mathbf{q}}-\left(\hat{\mathbf{q}}_{0} \cdot \hat{\mathbf{p}}\right)\left(\hat{\mathbf{q}}_{0} \cdot \hat{\mathbf{q}}\right)}{\sqrt{1-\left(\hat{\mathbf{q}}_{0} \cdot \hat{\mathbf{p}}\right)^{2}} \sqrt{1-\left(\hat{\mathbf{q}}_{0} \cdot \hat{\mathbf{q}}\right)^{2}}} \\
& =\frac{y_{p q}-x_{p} x_{q}}{\sqrt{1-x_{p}^{2}} \sqrt{1-x_{q}^{2}}}
\end{aligned}
$$

or

$$
y_{p q}=x_{p} x_{q}+\sqrt{1-x_{p}^{2}} \sqrt{1-x_{q}^{2}} x_{p q}^{q_{0}}
$$

For the special case where $\hat{\mathbf{q}}_{0}$ is parallel to the $z$-axis $\left(q_{0}\right.$-system $)$ one can write

$$
y_{p q}=x_{p} x_{q}+\sqrt{1-x_{p}^{2}} \sqrt{1-x_{q}^{2}} \cos \varphi_{p q}
$$

where the $\varphi_{p q}$ is the difference of the azimuthal angles of $\hat{\mathbf{p}}$ and $\hat{\mathbf{q}}$. However, the variable $\cos \varphi_{p q}$, which was used erroneously in [19] as third angular variable, is not rotationally invariant.

With the independent variables listed in Eq. (2.8) the matrix element of $\hat{T}$ is given as

$$
\left\langle\mathbf{p q}|\hat{T}| \varphi_{d} \mathbf{q}_{0}\right\rangle \equiv \hat{T}\left(p, x_{p}, x_{p q}^{q_{0}}, x_{q}, q ; q_{0}\right)
$$

Furthermore, $\hat{t}_{s}\left(\mathbf{p}^{\prime}, \mathbf{p}, z\right)$ is also a scalar function, and thus can be written in the form

$$
\hat{t}_{s}\left(\mathbf{p}^{\prime}, \mathbf{p}, z\right)=\hat{t}_{s}\left(p^{\prime}, p, \hat{\mathbf{p}}^{\prime} \cdot \hat{\mathbf{p}}, z\right)
$$

The most intricate dependence appears under the integral in Eq. (2.7) for the third angular variable of the $\hat{T}$ amplitude. According to Eq. 2.11 it is given as

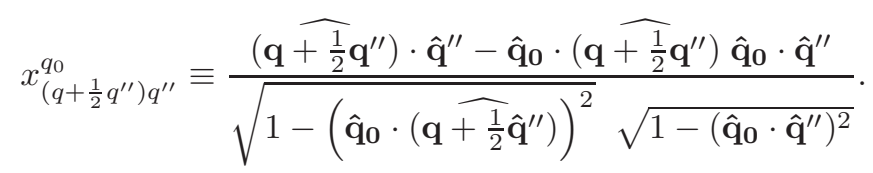


In view of the break-up singularities of the first denominator in Eq. (2.7) it is mandatory to choose the coordinate system for the q"-integration such that the z-axis points parallel to the vector $\hat{\mathbf{q}}$. Then one obtains for Eq. (2.16)

$$
x_{\left(q+\frac{1}{2} q^{\prime \prime}\right) q^{\prime \prime}}^{q_{0}}=\frac{\frac{q x^{\prime \prime}+\frac{1}{2} q^{\prime \prime}}{\sqrt{q^{2}+\frac{1}{4} q^{\prime \prime 2}+q q^{\prime \prime} x^{\prime \prime}}}-x_{q+\frac{1}{2} q^{\prime \prime}} x_{q^{\prime \prime}}}{\sqrt{1-x_{q+\frac{1}{2} q^{\prime \prime}}^{2}} \sqrt{1-x_{q^{\prime \prime}}^{2}}},
$$

where

$$
\begin{aligned}
x_{q^{\prime \prime}} & \equiv \hat{\mathbf{q}}^{\prime \prime} \cdot \hat{\mathbf{q}}_{\mathbf{o}}=x^{\prime \prime} x_{q}+\sqrt{1-x^{\prime \prime 2}} \sqrt{1-x_{q}^{2}} \cos \left(\varphi^{\prime \prime}-\varphi_{q_{0}}\right) \\
x_{q+\frac{1}{2} q^{\prime \prime}} & \equiv\left(\mathbf{q}+\frac{1}{2} \mathbf{q}^{\prime \prime}\right) \cdot \hat{\mathbf{q}}_{\mathbf{o}}=\frac{q x_{q}+\frac{1}{2} q^{\prime \prime} x_{q^{\prime \prime}}}{\sqrt{q^{2}+\frac{1}{4} q^{\prime \prime 2}+q q^{\prime \prime} x^{\prime \prime}}} .
\end{aligned}
$$

Here $\varphi_{q_{0}}$ is the azimuthal angle of $\hat{\mathbf{q}}_{0}$ in the coordinate system chosen for the q" -integration. These considerations lead to the explicit representation for the transition amplitude $\hat{T}$

$$
\begin{aligned}
& \hat{T}\left(p, x_{p}, x_{p q}^{q_{0}}, x_{q}, q ; q_{0}\right)=\varphi_{d}\left(\sqrt{q^{2}+\frac{1}{4} q_{0}^{2}+q q_{0} x_{q}}\right) \\
& \times \hat{t}_{s}\left(p, \sqrt{\frac{1}{4} q^{2}+q_{0}^{2}+q q_{0} x_{q}}, \frac{\frac{1}{2} q y_{p q}+q_{0} x_{p}}{\sqrt{\frac{1}{4} q^{2}+q_{0}^{2}+q q_{0} x_{q}}} ; E-\frac{3}{4 m} q^{2}\right) \\
& +\int_{0}^{\infty} d q^{\prime \prime} q^{\prime \prime 2} \int_{-1}^{+1} d x^{\prime \prime} \int_{0}^{2 \pi} d \varphi^{\prime \prime} \frac{1}{E-\frac{1}{m}\left(q^{2}+q q^{\prime \prime} x^{\prime \prime}+q^{\prime \prime 2}\right)+i \varepsilon} \\
& \times \hat{t}_{s}\left(p, \sqrt{\frac{1}{4} q^{2}+q^{\prime \prime 2}+q q^{\prime \prime} x^{\prime \prime}}, \frac{\frac{1}{2} q y_{p q}+q^{\prime \prime} y_{p q^{\prime \prime}}}{\sqrt{\frac{1}{4} q^{2}+q^{\prime \prime 2}+q q^{\prime \prime} x^{\prime \prime}}} ; E-\frac{3}{4 m} q^{2}\right) \\
& \times \frac{\hat{T}\left(\sqrt{q^{2}+\frac{1}{4} q^{\prime \prime 2}+q q^{\prime \prime} x^{\prime \prime}}, \frac{q x_{q}+\frac{1}{2} q^{\prime \prime} x_{q^{\prime \prime}}}{\sqrt{q^{2}+\frac{1}{4} q^{\prime \prime 2}+q q^{\prime \prime} x^{\prime \prime}}}, \frac{\frac{q x^{\prime \prime}+\frac{1}{2} q^{\prime \prime}}{\sqrt{q^{2}+\frac{1}{4} q^{\prime \prime 2}+q q^{\prime \prime} x^{\prime \prime}}}-x_{q+\frac{1}{2} q^{\prime \prime}} x_{q^{\prime \prime}}}{\sqrt{1-x_{q+\frac{1}{2} q^{\prime \prime}}} \sqrt{1-x_{q^{\prime \prime}}^{2}}}, x_{q^{\prime \prime}}, q^{\prime \prime} ; q_{0}\right)}{E-\frac{3}{4 m} q^{\prime \prime 2}-E_{d}+i \varepsilon}
\end{aligned}
$$

where in addition to Eq. (2.8) and the related variables of Eq. (2.12) and Eq. (2.18) the following variables occur

$$
\begin{aligned}
q^{\prime \prime} & =\left|\mathbf{q}^{\prime \prime}\right| \\
x^{\prime \prime} & =\hat{\mathbf{q}} \cdot \hat{\mathbf{q}}^{\prime \prime} \\
y_{p q^{\prime \prime}} & =\hat{\mathbf{p}} \cdot \hat{\mathbf{q}}^{\prime \prime}=y_{p q} x^{\prime \prime}+\sqrt{1-x^{\prime \prime 2}} \sqrt{1-y_{p q}^{2}} \cos \left(\varphi_{p}-\varphi^{\prime \prime}\right) .
\end{aligned}
$$

Like $\varphi_{q_{0}}$ in Eq. (2.18), the angle $\varphi_{p}$ in Eq. (2.20) is the azimuthal angle of $\hat{\mathbf{p}}$ in the $q$-system (i.e. the system where the z-axis is parallel to $\hat{\mathbf{q}}$ ). It remains to relate the angles $\varphi_{p}$ and $\varphi_{q_{0}}$ to the three angular variables $x_{p}, x_{q}$, and $x_{p q}^{q_{0}}$. As it is shown in Appendix A due to the $\varphi^{\prime \prime}$-integration, only the knowledge of $\cos \left(\varphi_{p}-\varphi_{q_{0}}\right)$ is required. Like cos $\varphi_{p q}$ in Eq. (2.13) is equal to $x_{p q}^{q_{0}}$ in the $q_{0}$-system, so is $\cos \left(\varphi_{p}-\varphi_{q_{0}}\right)$ equal to $x_{q_{0} p}^{q}$ in the $q$-system. Thus

$$
\cos \left(\varphi_{p}-\varphi_{q_{0}}\right)=x_{q_{0} p}^{q}=\frac{\hat{\mathbf{q}}_{0} \cdot \hat{\mathbf{p}}-\left(\hat{\mathbf{q}} \cdot \hat{\mathbf{q}}_{0}\right)(\hat{\mathbf{q}} \cdot \hat{\mathbf{p}})}{\sqrt{1-\left(\hat{\mathbf{q}} \cdot \hat{\mathbf{q}}_{0}\right)^{2}} \sqrt{1-(\hat{\mathbf{p}} \cdot \hat{\mathbf{q}})^{2}}}=\frac{x_{p}-x_{q} y_{p q}}{\sqrt{1-x_{q}^{2}} \sqrt{1-y_{p q}^{2}}}
$$

Because of that difference $\left(\varphi_{p}-\varphi_{q_{0}}\right)$, one can choose $\varphi_{q_{0}}$ arbitrarily, e.g. zero. Furthermore, $\cos \varphi_{p}$ and sin $\varphi_{p}$ required in Eq. 2.20 are given in terms of $\cos \left(\varphi_{p}-\varphi_{q_{0}}\right)$, as is shown in Appendix A This completes the definition of all relevant variables in Eq. (2.19). 


\section{AMPLITUDES AND CROSS SECTIONS FOR ELASTIC SCATTERING AND BREAK-UP PROCESSES}

The amplitude for elastic scattering is obtained by calculating the matrix element of the operator $U$ given in Eq. (2.3) as.

$$
\begin{aligned}
\left\langle\mathbf{q} \varphi_{d}|U| \mathbf{q}_{0} \varphi_{d}\right\rangle & =2 \varphi_{d}\left(\frac{1}{2} \mathbf{q}+\mathbf{q}_{0}\right)\left(E-\frac{1}{m}\left(q^{2}+\mathbf{q} \cdot \mathbf{q}_{0}+q_{0}^{2}\right)\right) \varphi_{d}\left(\mathbf{q}+\frac{1}{2} \mathbf{q}_{0}\right) \\
& +2 \int d^{3} q^{\prime \prime} \varphi_{d}\left(\frac{1}{2} \mathbf{q}+\mathbf{q}^{\prime \prime}\right) \frac{\left\langle\mathbf{q}+\frac{1}{2} \mathbf{q}^{\prime \prime}, \mathbf{q}^{\prime \prime}|\hat{T}| \mathbf{q}_{0} \varphi_{d}\right\rangle}{E-\frac{3}{4 m} q^{\prime \prime 2}-E_{d}+i \varepsilon}
\end{aligned}
$$

The amplitude for the full break-up process according to Eq. (2.2), is given by

$$
\left\langle\mathbf{p} \mathbf{q}\left|U_{0}\right| \mathbf{q}_{0} \varphi_{d}\right\rangle=\frac{\left\langle\mathbf{p q}|\hat{T}| \mathbf{q}_{0} \varphi_{d}\right\rangle}{E-\frac{3}{4 m} \mathbf{q}^{2}-E_{d}}+\frac{\left\langle-\frac{1}{2} \mathbf{p}+\frac{3}{4} \mathbf{q},-\mathbf{p}-\frac{1}{2} \mathbf{q}|\hat{T}| \mathbf{q}_{0} \varphi_{d}\right\rangle}{E-\frac{3}{4 m}\left(-\mathbf{p}-\frac{1}{2} \mathbf{q}\right)^{2}-E_{d}}+\frac{\left\langle-\frac{1}{2} \mathbf{p}-\frac{3}{4} \mathbf{q},+\mathbf{p}-\frac{1}{2} \mathbf{q}|\hat{T}| \mathbf{q}_{0} \varphi_{d}\right\rangle}{E-\frac{3}{4 m}\left(+\mathbf{p}-\frac{1}{2} \mathbf{q}\right)^{2}-E_{d}}
$$

The equation for the elastic operator $U$ follows from Eqs. (2.1) and (2.3). It is given as

$$
U|\phi\rangle=P G_{0}^{-1}|\phi\rangle+P t G_{0} U|\phi\rangle
$$

Straightforward and well known steps [5] based on this equation lead to the unitarity relation

$$
\begin{aligned}
\left\langle\phi|U| \phi^{\prime}\right\rangle^{*}-\left\langle\phi^{\prime}|U| \phi\right\rangle & =\int d^{3} q\left\langle\phi_{q}|U| \phi^{\prime}\right\rangle^{*} 2 \pi i \delta\left(E-E_{d}-\frac{3}{4 m} q^{2}\right)\left\langle\phi_{q}|U| \phi\right\rangle \\
& +\frac{1}{3} \int d^{3} p d^{3} q\left\langle\phi_{0}\left|U_{0}\right| \phi^{\prime}\right\rangle^{*} 2 \pi i \delta\left(E-\frac{p^{2}}{m}-\frac{3}{4 m} q^{2}\right)\left\langle\phi_{0}\left|U_{0}\right| \phi\right\rangle
\end{aligned}
$$

We want to point out that there is a misprint in Eq. (202) of Ref. [5], the factor 1/3 is missing.

Using the variables defined in the previous section, and having in mind that for elastic scattering $|\mathbf{q}|=|\mathbf{q o}|$, the amplitude for elastic scattering according to Eq. (3.1) can be expressed as

$$
\begin{aligned}
& \left\langle\mathbf{q} \varphi_{d}|U| \mathbf{q}_{0} \varphi_{d}\right\rangle \equiv U\left(q_{0}, x_{q}\right)=2 \varphi_{d}^{2}\left(q_{0} \sqrt{\frac{5}{4}+x_{q}}\right)\left(E-\frac{q_{0}^{2}}{m}\left(2+x_{q}\right)\right) \\
& +2 \int_{0}^{\infty} d q^{\prime \prime} q^{\prime \prime 2} \int_{-1}^{+1} d x^{\prime \prime} \int_{0}^{2 \pi} d \varphi^{\prime \prime} \frac{1}{E-\frac{3}{4 m} q^{\prime \prime 2}-E_{d}+i \varepsilon} \\
& \times \varphi_{d}\left(\sqrt{\frac{1}{4} q_{0}^{2}+q^{\prime \prime 2}+q_{0} q^{\prime \prime} y_{q q^{\prime \prime}}}\right) \\
& \times \hat{T}\left(\sqrt{q_{0}^{2}+\frac{1}{4} q^{\prime \prime 2}+q_{0} q^{\prime \prime} y_{q q^{\prime \prime}}}, \frac{q_{0} x_{q}+\frac{1}{2} q^{\prime \prime} y_{q_{0} q^{\prime \prime}}}{\sqrt{q_{0}^{2}+\frac{1}{4} q^{\prime \prime 2}+q_{0} q^{\prime \prime} y_{q q^{\prime \prime}}}}, \frac{\frac{q_{0} y_{q q^{\prime \prime}}+\frac{1}{2} q^{\prime \prime}}{\sqrt{q_{0}^{2}+\frac{1}{4} q^{\prime \prime 2}+q_{0} q^{\prime \prime} y_{q q^{\prime \prime}}}}-x_{\pi_{p}} x_{\pi_{q}}}{\sqrt{1-x_{\pi_{p}}^{2}} \sqrt{1-x_{\pi_{q}}^{2}}}, y_{q_{0} q^{\prime \prime}}, q^{\prime \prime} ; q_{0}\right)
\end{aligned}
$$

with

$$
\begin{aligned}
y_{q q^{\prime \prime}} & =\hat{\mathbf{q}} \cdot \hat{\mathbf{q}}^{\prime \prime} \\
y_{q_{0} q^{\prime \prime}} & =\hat{\mathbf{q}}_{0} \cdot \hat{\mathbf{q}}^{\prime \prime}=x_{\pi_{q}} \\
x_{\pi_{p}} & =\frac{q_{0} x_{q}+\frac{1}{2} q^{\prime \prime} y_{q_{0} q^{\prime \prime}}}{\sqrt{q_{0}^{2}+\frac{1}{4} q^{\prime \prime 2}+q_{0} q^{\prime \prime} y_{q q^{\prime \prime}}}}
\end{aligned}
$$

At this point, the choice of a specific coordinate system for the $\mathbf{q}^{\prime \prime}$-integration is still open. The angular variable $x_{q}=\hat{\mathbf{q}} \cdot \hat{\mathbf{q}} \mathbf{0}$ represents the scattering angle. If the $\mathrm{z}$-axis is chosen parallel to $\hat{\mathbf{q}}_{\mathbf{0}}$, the angles are

$$
\begin{aligned}
y_{q q^{\prime \prime}} & =x_{q} x^{\prime \prime}+\sqrt{1-x_{q}^{2}} \sqrt{1-x^{\prime \prime 2}} \cos \left(\varphi_{q}-\varphi^{\prime \prime}\right) . \\
y_{q_{0} q^{\prime \prime}} & =x^{\prime \prime}
\end{aligned}
$$


While having the z-axis parallel to $\hat{\mathbf{q}}_{0}$ may be the intuitive choice to describe the scattering with a given beam direction, we can in principle also choose the z-axis parallel to $\hat{\mathbf{q}}$. In that case the angels in Eq. 3.6. are given by

$$
\begin{aligned}
y_{q q^{\prime \prime}} & =x^{\prime \prime} \\
y_{q_{0} q^{\prime \prime}} & =x_{q} x^{\prime \prime}+\sqrt{1-x_{q}^{2}} \sqrt{1-x^{\prime \prime 2}} \cos \left(\varphi_{q_{0}}-\varphi^{\prime \prime}\right) .
\end{aligned}
$$

The elastic cross section depends on the angle between the vectors $\hat{\mathbf{q}}_{\mathbf{0}}$ and $\hat{\mathbf{q}}$, but not on the choice of z-axis. We use the possibility of calculating the matrix elements of $U$ in the two different coordinate systems to test the quality of our numerical calculations.

The differential elastic cross section in the c.m. frame is given by

$$
\frac{d \sigma_{e l}}{d \Omega}=\left(\frac{2 m}{3}\right)^{2}(2 \pi)^{4}\left|U\left(q_{0} x_{q}\right)\right|^{2}
$$

and the corresponding total elastic cross section is

$$
\sigma_{e l}=\int d \Omega \frac{d \sigma_{e l}}{d \Omega}=\left(\frac{2 m}{3}\right)^{2}(2 \pi)^{5} \int_{-1}^{+1} d x \|\left. U\left(q_{0}, x\right)\right|^{2} .
$$

The full break-up amplitude is given in Eq. (3.2). On the energy shell $p$ and $q$ are constrained by $p^{2}+\frac{3}{4} q^{2}=m E$. As function of all five variables and the projectile momentum it reads

$$
U_{0}\left(p, x_{p}, x_{p q}^{q_{0}}, x_{q}, q, q_{0}\right)=\frac{\hat{T}\left(p, x_{p}, x_{p q}^{q_{0}}, x_{q}, q, q_{0}\right)}{E-\frac{3}{4 m} q^{2}-E_{d}}+\frac{\hat{T}\left(p_{2}, x_{p_{2}}, x_{p_{2} q_{2}}^{q_{0}}, x_{q_{2}}, q_{2}, q_{0}\right)}{E-\frac{3}{4 m} q_{2}^{2}-E_{d}}+\frac{\hat{T}\left(p_{3}, x_{p_{3}}, x_{\left.p_{3} q_{3}, x_{q_{3}}, q_{3}, q_{0}\right)}^{E} . \frac{3}{4 m} q_{3}^{2}-E_{d}\right.}{E .}
$$

Here the variables are defined as

$$
\begin{aligned}
& y_{p q}=x_{p} x_{q}+\sqrt{1-x_{p}^{2}} \sqrt{1-x_{q}^{2}} x_{p q}^{q_{0}} \\
& p_{2}=\left|-\frac{1}{2} \mathbf{p}+\frac{3}{4} \mathbf{q}\right|=\frac{1}{2} \sqrt{p^{2}+\frac{9}{4} q^{2}-3 p q y_{p q}} \\
& q_{2}=\left|-\mathbf{p}-\frac{1}{2} \mathbf{q}\right|=\sqrt{p^{2}+\frac{1}{4} q^{2}+p q y_{p q}} \\
& p_{3}=\left|-\frac{1}{2} \mathbf{p}-\frac{3}{4} \mathbf{q}\right|=\frac{1}{2} \sqrt{p^{2}+\frac{9}{4} q^{2}+3 p q y_{p q}} \\
& q_{3}=\left|+\mathbf{p}-\frac{1}{2} \mathbf{q}\right|=\sqrt{p^{2}+\frac{1}{4} q^{2}-p q y_{p q}} \\
& x_{p_{2}}=\hat{\mathbf{p}}_{2} \cdot \hat{\mathbf{q}}_{0}=\frac{-\frac{1}{2} p x_{p}+\frac{3}{4} q x_{q}}{p_{2}} \\
& x_{q_{2}}=\hat{\mathbf{q}}_{2} \cdot \hat{\mathbf{q}}_{0}=\frac{-p x_{p}-\frac{1}{2} q x_{q}}{q_{2}} \\
& x_{p_{3}}=\hat{\mathbf{p}}_{3} \cdot \hat{\mathbf{q}}_{0}=\frac{-\frac{1}{2} p x_{p}-\frac{3}{4} q x_{q}}{p_{3}} \\
& x_{q_{3}}=\hat{\mathbf{q}}_{3} \cdot \hat{\mathbf{q}}_{0}=\frac{+p x_{p}-\frac{1}{2} q x_{q}}{q_{3}} \\
& x_{p_{2} q_{2}}^{q_{0}}=\left(\mathbf{q}_{0} \widehat{\times} \mathbf{p}_{2}\right) \cdot\left(\mathbf{q}_{0} \widehat{\times} \mathbf{q}_{2}\right)=\frac{\frac{\frac{1}{2} p^{2}-\frac{3}{8} q^{2}-\frac{1}{2} p q y_{p q}}{p_{2} q_{2}}-x_{p_{2}} x_{q_{2}}}{\sqrt{1-x_{p_{2}}^{2}} \sqrt{1-x_{q_{2}}^{2}}} \\
& x_{p_{3} q_{3}}^{q_{0}}=\left(\mathbf{q}_{0} \widehat{\times} \mathbf{p}_{3}\right) \cdot\left(\mathbf{q}_{0} \widehat{\times} \mathbf{q}_{3}\right)=\frac{\frac{-\frac{1}{2} p^{2}+\frac{3}{8} q^{2}-\frac{1}{2} p q y_{p q}}{p_{3} q_{3}}-x_{p_{3}} x_{q_{3}}}{\sqrt{1-x_{p_{3}}^{2}} \sqrt{1-x_{q_{3}}^{2}}}
\end{aligned}
$$

The five-fold differential break-up cross section is given in the c.m. frame

$$
\frac{d^{5} \sigma_{b r}}{d \Omega_{p} d \Omega_{q} d q}=\frac{(2 \pi)^{4} m^{2}}{3 q_{0}} q^{2} \sqrt{m E-\frac{3}{4} q^{2}}\left|U_{0}\left(p, x_{p}, x_{p q}^{q_{0}}, x_{q}, q, q_{0}\right)\right|^{2}
$$


It is convenient to calculate the total break-up cross section in the c.m. frame, since there are no kinematic restrictions on the relative angles. For the explicit calculation we can make different choices of the z-axis, e.g. it can be parallel to the direction $\hat{\mathbf{q}}_{\mathbf{0}}$ of the projectile, or parallel to either one of the Jacobi vectors $\hat{\mathbf{q}}$ and $\hat{\mathbf{p}}$. The different choices will obviously result in different angular integrations. For completeness we give all three choices here. This will be used as a highly non-trivial test of the numerical results, as will be demonstrated in the next Section. If the z-axis is parallel to $\hat{\text { qo }}$ we have

$$
\int d \Omega_{p} d \Omega_{q}=2 \pi \int_{-1}^{+1} d x_{p}^{\prime \prime} \int_{-1}^{+1} d x_{q}^{\prime \prime} \int_{0}^{2 \pi} d \varphi_{p q}^{\prime \prime}
$$

with

$$
x_{p} \rightarrow x_{p}^{\prime \prime}, \quad x_{q} \rightarrow x_{q}^{\prime \prime}, \quad x_{p q}^{q_{0}} \rightarrow \cos \varphi_{p q}^{\prime \prime}
$$

If the z-axis is parallel to $\hat{\mathbf{q}}$, the angular integration becomes

$$
\int d \Omega_{p} d \Omega_{q}=2 \pi \int_{-1}^{+1} d x_{q}^{\prime \prime} \int_{-1}^{+1} d y_{p q}^{\prime \prime} \int_{0}^{2 \pi} d \varphi_{p q_{0}}^{\prime \prime}
$$

with

$$
\begin{aligned}
x_{p} & \rightarrow x_{q}^{\prime \prime} y_{p q}^{\prime \prime}+\sqrt{1-x_{q}^{\prime \prime 2}} \sqrt{1-y_{p q}^{\prime \prime 2}} \cos \varphi_{p q_{0}}^{\prime \prime}, \\
x_{q} & \rightarrow x_{q}^{\prime \prime}, \\
x_{p q}^{q_{0}} & \rightarrow \frac{y_{p q}^{\prime \prime}-\left(x_{q}^{\prime \prime} y_{p q}^{\prime \prime}+\sqrt{1-x_{q}^{\prime \prime 2}} \sqrt{1-y_{p q}^{\prime \prime 2}} \cos \varphi_{p q_{0}}^{\prime \prime}\right) x_{q}^{\prime \prime}}{\sqrt{1-\left(x_{q}^{\prime \prime} y_{p q}^{\prime \prime}+\sqrt{1-x_{q}^{\prime \prime 2}} \sqrt{1-y_{p q}^{\prime \prime 2}} \cos \varphi_{p q_{0}}^{\prime \prime}\right)^{2}} \sqrt{1-x_{q}^{\prime \prime 2}}} .
\end{aligned}
$$

Finally, if the z-axis is parallel to $\hat{\mathbf{p}}$, the angular integration is

$$
\int d \Omega_{p} d \Omega_{q}=2 \pi \int_{-1}^{+1} d x_{p}^{\prime \prime} \int_{-1}^{+1} d y_{p q}^{\prime \prime} \int_{0}^{2 \pi} d \varphi_{q q_{0}}^{\prime \prime}
$$

with

$$
\begin{aligned}
x_{p} & \rightarrow x_{p}^{\prime \prime}, \\
x_{q} & \rightarrow x_{p}^{\prime \prime} y_{p q}^{\prime \prime}+\sqrt{1-x_{p}^{\prime \prime 2}} \sqrt{1-y_{p q}^{\prime \prime 2}} \cos \varphi_{q q_{0}}^{\prime \prime}, \\
x_{p q}^{q_{0}} & \rightarrow \frac{y_{p q}^{\prime \prime}-\left(x_{p}^{\prime \prime} y_{p q}^{\prime \prime}+\sqrt{1-x_{p}^{\prime \prime 2}} \sqrt{1-y_{p q}^{\prime \prime 2}} \cos \varphi_{q q_{0}}^{\prime \prime}\right) x_{p}^{\prime \prime}}{\sqrt{1-\left(x_{p}^{\prime \prime} y_{p q}^{\prime \prime}+\sqrt{1-x_{p}^{\prime \prime 2}} \sqrt{1-y_{p q}^{\prime \prime 2}} \cos \varphi_{q q_{0}}^{\prime \prime}\right)^{2}} \sqrt{1-x_{p}^{\prime \prime 2}}} .
\end{aligned}
$$

Let us define a function $\mathcal{F}(p, q)$ as

$$
\mathcal{F}(p, q)=\int d \Omega_{p} d \Omega_{q}\left|\left\langle\phi_{0}\left|U_{0}\right| \phi\right\rangle\right|^{2}
$$

where the angle integrations over the break-up amplitude is carried out. This function should be independent of the coordinate system in which the angle integrations are performed. We use this property to check our numerical calculations. This is a non-trivial test of our calculation, since especially at higher energies the transition amplitude $\hat{T}$ develops stronger angle dependencies, which challenge the accuracy of the multi-dimensional interpolations.

The angle integrated break-up cross section is given as

$$
\frac{d \sigma_{b r}}{d q}=\frac{1}{3} \frac{(2 \pi)^{4} m^{2}}{3 q_{0}} q^{2} \sqrt{m E-\frac{3}{4} q^{2}} \mathcal{F}\left(\sqrt{m E-\frac{3}{4} q^{2}}, q\right),
$$

and the total break-up cross section reads

$$
\sigma_{b r}=\frac{1}{3} \frac{(2 \pi)^{4} m^{2}}{3 q_{0}} \int_{0}^{\sqrt{\frac{4 m E}{3}}} d q q^{2} \sqrt{m E-\frac{3}{4} q^{2}} \mathcal{F}\left(\sqrt{m E-\frac{3}{4} q^{2}}, q\right) .
$$


Using now the unitarity relation from Eq. (3.4) the optical theorem gives

$$
\sigma_{t o t}=\sigma_{e l}+\sigma_{b r}=-\frac{4 m(2 \pi)^{3}}{3 q_{0}} \operatorname{Im} U\left(q_{0}, 1\right) .
$$

For later use we also mention the semi-exclusive cross section, where only one particle is detected in the break-up process

$$
\frac{d \sigma}{d \Omega_{q} d q}=(2 \pi)^{4} \frac{m^{2}}{3 q_{0}} p q^{2} \int d \hat{p}\left|U_{0}\left(p, x_{p}, x_{p q}^{q_{0}}, x_{q}, q, q_{0}\right)\right|^{2}
$$

\section{NUMERICAL METHODS}

The fully off-shell two-body t-matrix $t\left(\mathbf{p}^{\prime}, \mathbf{p}, z\right)$ is solved directly from the Lippmann-Schwinger equation as function of its vector variables [20] for the off-shell energies $E-\frac{3}{4 m} q^{2}$ as required by Eq. (2.4). The Faddeev equation is iterated, generating the multiple scattering series, which is then summed by the Padé method [21, 22]. We use it in the form of a continued fraction expansion as layed out in Ref. [18].

The first integration to be performed in solving Eq. (2.19) by iteration is the integration over the azimuthal angle $\varphi^{\prime \prime}$. This leads to a function of variables, $q^{\prime \prime}$ and $x^{\prime \prime}$ and requires interpolation in the second and third arguments of $t_{s}$ and the first four arguments of $\hat{T}$. Our spline interpolations are based on the cubic Hermite splines given in [23].

Let $F\left(q^{\prime \prime}, x^{\prime \prime}\right)$ be the resulting function in each step of the iteration. Clearly, it depends in addition on the fixed variables $p, q, x_{p}, x_{q}$, and $x_{p q}^{q_{0}}$, which are omitted for clarity. Then the next task is performing the remaining two singular integrations,

$$
I=\int_{0}^{\infty} d q^{\prime \prime} q^{\prime \prime 2} \int_{-1}^{+1} d x^{\prime \prime} \frac{F\left(q^{\prime \prime}, x^{\prime \prime}\right)}{\left(E-\frac{1}{m}\left(q^{2}+q^{\prime \prime 2}+q q^{\prime \prime} x^{\prime \prime}\right)+i \varepsilon\right)\left(E-\frac{3}{4 m} q^{\prime \prime 2}-E_{d}+i \varepsilon\right)}
$$

If the c.m. energy $E$ is below the three-body break-up threshold, only the second denominator is singular, and the simple pole can be treated by standard subtraction methods.

The intricate problem arises above the three-body break-up threshold, when in addition the first denominator can vanish. It has the form

$$
\frac{1}{E-\frac{1}{m}\left(q^{2}+q q^{\prime \prime} x^{\prime \prime}+q^{\prime \prime 2}\right)+i \varepsilon}=\frac{\frac{m}{q q^{\prime \prime}}}{x_{0}-x^{\prime \prime}+i \varepsilon}
$$

with

$$
x_{0}=\frac{m E-q^{2}-q^{\prime \prime 2}}{q q^{\prime \prime}} .
$$

For $\left|x_{0}\right| \leq 1$ a so-called moving singularity arises in the $q^{\prime \prime}$ - $x^{\prime \prime}$-integration, since $x_{0}$ depends on $q$. The direct treatment of those moving singularities using real variables has been discussed in the literature [2]. We briefly review the appearance of these singularities in form of logarithms, since we introduce a new quasi-analytic integration based on Spline functions. The condition $\left|x_{0}\right|=1$ leads to the pole positions

$$
q^{\prime \prime}= \pm \frac{q}{2} \pm \sqrt{m E-\frac{3}{4} q^{2}},
$$

and one arrives at the well known shape in the $q$ - $q^{\prime \prime}$-plane for $\left|x_{0}\right| \leq 1$ shown in Fig. 2 . This region is bounded by

$$
q_{+}=\frac{q}{2}+\sqrt{Q_{0}^{2}-\frac{3}{4} q^{2}}
$$

and

$$
q_{-}= \begin{cases}-\frac{q}{2}+\sqrt{Q_{0}^{2}-\frac{3}{4} q^{2}} & q<Q_{0} \\ +\frac{q}{2}-\sqrt{Q_{0}^{2}-\frac{3}{4} q^{2}} & q>Q_{0}\end{cases}
$$


where $Q_{0}=\sqrt{m E}$. Apparently there is no singularity if $q>q_{\max } \equiv \sqrt{\frac{4 m}{3} E}$. We distinguish four cases, $q=0$, $0<q<q_{\max }, q=q_{\max }$, and $q>q_{\max }$. The case $q=0$ reduces to a simple subtraction and will not be discussed. For $0<q<q_{\max }$ we only consider the part of the $q^{\prime \prime}$-integration which contains the moving singularities. It has the schematic form

$$
I^{\prime}=\int_{0}^{q_{\max }} d q^{\prime \prime} \int_{-1}^{+1} d x^{\prime \prime} \frac{f\left(q^{\prime \prime}, x^{\prime \prime}\right)}{x_{0}-x^{\prime \prime}+i \varepsilon}
$$

The first step is to perform a subtraction of the pole, which we carry out in the entire square $0 \leq q, q^{\prime \prime} \leq q_{\max }$ by defining

$$
\hat{f}\left(q^{\prime \prime}, x_{0}\right)=\left\{\begin{array}{cl}
f\left(q^{\prime \prime}, x_{0}\right): & \left|x_{0}\right| \leq 1 \\
f\left(q^{\prime \prime}, \frac{x_{0}}{\left|x_{0}\right|}\right): & \left|x_{0}\right|>1
\end{array}\right.
$$

We obtain

$$
\begin{aligned}
I^{\prime} & =\int_{0}^{q_{\max }} d q^{\prime \prime} \int_{-1}^{+1} d x^{\prime \prime} \frac{f\left(q^{\prime \prime}, x^{\prime \prime}\right)-\hat{f}\left(q^{\prime \prime}, x_{0}\right)}{x_{0}-x^{\prime \prime}} \\
& +\int_{0}^{q_{\max }} d q^{\prime \prime} \hat{f}\left(q^{\prime \prime}, x_{0}\right) \ln \left|\frac{1+x_{0}}{1-x_{0}}\right|-i \pi \int_{0}^{q_{\max }} d q^{\prime \prime} \Theta\left(1-\left|x_{0}\right|\right) \hat{f}\left(q^{\prime \prime}, x_{0}\right) .
\end{aligned}
$$

Now we define $q_{-}=\frac{q}{2}-\sqrt{Q_{0}^{2}-\frac{3}{4} q^{2}}$ and obtain

$$
\ln \left|\frac{1+x_{0}}{1-x_{0}}\right|=\left(-\frac{q_{-}}{\left|q_{-}\right|} \ln \left|q^{\prime \prime}+\right| q_{-}||-\ln \left|q^{\prime \prime}+q_{+}\right|\right)+\left(+\frac{q_{-}}{\left|q_{-}\right|} \ln \left|q^{\prime \prime}-\right| q_{-}||+\ln \left|q^{\prime \prime}-q_{+}\right|\right) .
$$

This leads to the well separated part of the integral which contains the logarithmic singularity

$$
\int_{0}^{q_{\max }} d q^{\prime \prime} \hat{f}\left(q^{\prime \prime}, x_{0}\right)\left(+\frac{q_{-}}{\left|q_{-}\right|} \ln \left|q^{\prime \prime}-\right| q_{-}||+\ln \left|q^{\prime \prime}-q_{+}\right|\right) .
$$

It is here that we introduce the new technique that relies on cubic spline integration.

We divide the range of integration $\left[0, q_{\max }\right]$ into intervals bounded by a set of grid points $q_{i}$. The set of grid points is supposed to be dense enough to interpolate the function $\hat{f}\left(q^{\prime \prime}, x_{0}\right) \equiv f\left(q^{\prime \prime}\right)$ sufficiently well by cubic Hermitean splines [23]. In Ref. 23] a detailed presentation of these spline functions is given specific for our use. For the convenience of the reader we now switch to the notation of [23] and denote the end points of the $i$-th interval by $x_{1}$ and $x_{2}$ and the two adjacent grid points to the left and right of the $i$-th interval by $x_{0}$ and $x_{3}$, respectively. Then as detailed in [23] the interpolating function in the $i$-th interval (replacing $f\left(q^{\prime \prime}\right) \equiv f(x)$ ) can be written as

$$
f_{i}(x)=\sum_{j=0}^{3} S_{j}(x) f\left(x_{j}\right),
$$

where the modified spline functions are

$$
\begin{aligned}
S_{0}(x) & =-\phi_{3}(x) \frac{x_{2}-x_{1}}{x_{1}-x_{0}} \frac{1}{x_{2}-x_{0}}, \\
S_{1}(x) & =\phi_{1}(x)+\phi_{3}\left(\frac{x_{2}-x_{1}}{x_{1}-x_{0}}-\frac{x_{1}-x_{0}}{x_{2}-x_{1}}\right) \frac{1}{x_{2}-x_{0}}-\phi_{4}(x) \frac{x_{3}-x_{2}}{x_{2}-x_{1}} \frac{1}{x_{3}-x_{1}}, \\
S_{2}(x) & =\phi_{2}(x)+\phi_{3} \frac{x_{1}-x_{0}}{x_{2}-x_{1}} \frac{1}{x_{2}-x_{0}}+\phi_{4}(x)\left(\frac{x_{3}-x_{2}}{x_{2}-x_{1}}-\frac{x_{2}-x_{1}}{x_{3}-x_{2}}\right) \frac{1}{x_{3}-x_{1}}, \\
S_{3}(x) & =\phi_{4}(x) \frac{x_{2}-x_{1}}{x_{3}-x_{2}} \frac{1}{x_{3}-x_{1}} .
\end{aligned}
$$

with

$$
\phi_{1}(x)=\frac{\left(x_{2}-x\right)^{2}}{\left(x_{2}-x_{1}\right)^{3}}\left[\left(x_{2}-x_{1}\right)+2\left(x-x_{1}\right)\right]
$$




$$
\begin{aligned}
& \phi_{2}(x)=\frac{\left(x_{1}-x\right)^{2}}{\left(x_{2}-x_{1}\right)^{3}}\left[\left(x_{2}-x_{1}\right)+2\left(x_{2}-x\right)\right], \\
& \phi_{3}(x)=\frac{\left(x-x_{1}\right)\left(x_{2}-x\right)^{2}}{\left(x_{2}-x_{1}\right)^{2}}, \\
& \phi_{4}(x)=\frac{\left(x-x_{1}\right)^{2}\left(x-x_{2}\right)}{\left(x_{2}-x_{1}\right)^{2}} .
\end{aligned}
$$

Therefore, in view of Eqs. (4.11)- (4.14), the following integrals occur for $i=1 \cdots 4$

$$
\begin{aligned}
& \bar{\phi}_{1}=\int_{x_{i}}^{x_{i+1}} \phi_{1} \ln |x-q| d x, \\
& \bar{\phi}_{2}=\int_{x_{i}}^{x_{i+1}} \phi_{2} \ln |x-q| d x, \\
& \bar{\phi}_{3}=\int_{x_{i}}^{x_{i+1}} \phi_{3} \ln |x-q| d x, \\
& \bar{\phi}_{4}=\int_{x_{i}}^{x_{i+1}} \phi_{4} \ln |x-q| d x .
\end{aligned}
$$

with $q=\left|q_{-}\right|, q_{+}$. Consequently the five different cases $q<x_{i}<x_{i+1}, q=x_{i}<x_{i+1}, x_{i}<q<x_{i+1}, x_{i}<q=x_{i+1}$, and $x_{i}<x_{i+1}<q$ occur. Since the functions $\phi_{i}(x)$ are cubic polynomials, the integrals in Eq. (4.15) can be performed analytically. We leave the explicit calculation to interested practitioner and refer to 24] for a detailed presentation. According to our experience that manner to integrate the moving logarithmic singularities is a very good alternative to the more common subtraction method [2].

Finally, for $q=q_{\max }$ we also apply the subtraction over the extended region $0 \leq q^{\prime \prime} \leq q_{\max }$. In that case $q_{+}=q_{-}=\frac{q_{\max }}{2}$ and when $q^{\prime \prime}=\frac{q_{\max }}{2}$ then $x_{0}=-1$. Analogous steps lead to that part of the integral, which contains the logarithmic singularity,

$$
\int_{0}^{q_{\max }} d q^{\prime \prime} \hat{f}\left(q^{\prime \prime},-1\right) \ln \left|q^{\prime \prime}-\frac{q_{\max }}{2}\right|
$$

which is again evaluated by spline based integration.

In order to test the correctness as well as the accuracy of our calculations we carried out a variety of numerical tests. Unfortunately we could not compare to work by other groups, since to the best of our knowledge no comparable work at higher energies exists.

Apart from the projectile momentum $q_{0}$, the amplitude $\hat{T}$ of Eq. (2.19) depends on five variables $p, x_{p}, x_{p q}^{q_{0}}, x_{q}$, and $q$. In addition, there are the integration variables $q^{\prime \prime}, x^{\prime \prime}$ and $\varphi^{\prime \prime}$. All calculations listed are based on the MalflietTjon type potential which is explicitly given in the next section. The fully off-shell two-body $t$-matrix, $t\left(p^{\prime}, p, x, \varepsilon\right)$, is obtained for each fixed energy on a symmetric momentum grid with $60 p\left(p^{\prime}\right)$ points and $40 x$ points. Since the momentum region which contributes to a solution of the two-body $t$-matrix is quite different from the region of importance in a three-body calculation, we map our solution for $t_{\mathrm{s}}$ onto the momentum grid relevant for the three-body transition amplitude. This is done by applying the Lippmann-Schwinger equation repeatedly. The $t$ matrix $t_{\mathrm{s}}\left(p^{\prime}, p, x, \varepsilon\right)$ is obtained at energies $\varepsilon=E-\frac{3}{4 m} q^{2}$, exactly at the $q$ values needed in the three-body transition amplitude of Eq. (2.19).

In carrying out our calculations, it turns out that there are essentially two separate issues governing the quality of the results. The first is the angle dependence of the transition amplitude of Eq. (2.19). It is to be expected that the angle dependence is weak at low energies and increases with higher energies, reflecting the need to include more and more partial waves at higher energies in a partial wave based calculations. As example we list in Table \ the elastic and break-up total cross sections together with the total cross section extracted from the imaginary part of $U$ in forward direction, Eq. (3.23). At $0.01 \mathrm{GeV} 12$ points for all angles are clearly sufficient, whereas at $0.1 \mathrm{GeV}$ this is not so. Table पlists the elastic, break-up and total cross section as function of the angle variables, and we see that one needs at least 16 points for all angles. At $0.5 \mathrm{GeV}$ we find that the biggest angular dependence occurs in $x_{q}$ and $x "$, and the least dependence in the azimuthal angle $\varphi$ " and the angle $x_{p q}^{q_{0}}$, and take this into account in our choice of angle points.

The other issue is the quality of the calculation in the singular regime, i.e. in the integration region bounded by $q_{\max }$ in Fig. 2 We divide the integration grid for $q$ " into the intervals $\left(0, q_{\max }\right) \cup\left(q_{\max }, \bar{q}\right)$, where $q_{\max }=\sqrt{\frac{4 m}{3} E}$ and $\bar{q}=20 \mathrm{fm}^{-1}$. The inteval boundaries 0 and $q_{\max }$ are handled explicitly. As the energy increases, $q_{\max }$ increases, 
and we need to take this into account by changing the distribution of the $q$ " points as function of energy within the different $q^{\prime \prime}$-intervals, i.e. put more points into the interval $\left(0, q_{\max }\right)$ and less into $\left(q_{\max }, \bar{q}\right)$. From the number of points in $\left(0, q_{\max }\right)$ one can define an average point distance $\Delta_{q} \equiv q_{\max } /\left(\right.$ number of points in $\left.\left(0, q_{\max }\right)\right)$ in this interval. In Fig. 3. 3 we show the dependence of the calculation on $\Delta_{q}$ by using the percent error $\delta_{\text {opt }}=\left|\sigma_{\text {opt }}-\sigma_{\text {el }}-\sigma_{b r}\right| / \sigma_{o p t} \times 100$ in the fulfillment of the optical theorem as quality measure. At $0.01 \mathrm{GeV}$ it is quite easy to make the average point distance very small in the interval $\left(0, q_{\max }\right)$, since $q_{\max }$ is only $75 \mathrm{MeV}$. The top panel of Fig. 3 shows that the percent error $\delta_{\text {opt }}$ drops linearly below $0.1 \%$ and flattens out at $\Delta_{q}=3.5 \mathrm{MeV}$ where most likely errors in the interpolation start to play a role. At projectile energy $0.1 \mathrm{GeV} q_{\max }$ is already $284 \mathrm{MeV}$, and $\Delta_{q}$ is naturally much larger with a reasonable number of $q^{\prime \prime}$ points. The dependence of the $\delta_{\text {opt }}$ for $0.1 \mathrm{GeV}$ on $\Delta_{q}$ is shown in the middle panel of Fig. 3 for two different cases. An angular grid size of 12 points is indicated by the open squares, one of 24 points by crosses. A comparison of the calculations shows at $\Delta_{q}=15 \mathrm{MeV}$ the calculation with 12 angular points can not be improved any further, the calculations start to oscillate for smaller $\Delta_{q}$, accidentally giving a very good agreement at $\Delta_{q}=13 \mathrm{MeV}$. Increasing the number of angle points to 24 shows a further linear decrease in the error (cross symbols) into the $1 \%$ region of $\delta_{\text {opt }}$ at a $\Delta_{q}=9 \mathrm{MeV}$. This is consistent with the findings shown in the top panel. We continue to study the dependence of $\Delta_{q}$ at $0.5 \mathrm{GeV}$, where $q_{\max }=644 \mathrm{MeV}$. Here we immediately use 24 angle points as suggested from Table \A total of 30 integration points in $\left(0, q_{\max }\right)$ leads to $\Delta_{q}=22 \mathrm{MeV}$ and $\delta_{\text {opt }} \approx 10 \%$, which is consistent with values in the middle panel. The $10 \%$ error is also consistent with the value for the total break-up cross section in the last row of Table \. which indicates that $\sigma_{b r}$ is not yet converged. From the systematics at the different energies shown in Fig. 3 we can extrapolate on the $\Delta_{q}$ needed to reduce the error in the optical theorem. Due to computer time limitations we have not pushed this any further.

In Table I the total elastic, the total breakup as well as the total cross section evaluated according to Eq. (3.23) via the optical theorem are given and shown as function of various sets of grid points. The momentum grids for $p$ and $q$ are discretized with 49 points each. The integration variable $q^{\prime \prime}$ plays the same role as $q$, and is therefore also discretized with 49 points distributed over the intervals $\left(0, q_{\max }\right) \cup\left(q_{\max }, \bar{q}\right)$ in an energy dependent way according to the insights described above. The values given in last row of each energy correspond to the points at the smallest $\Delta_{q}$ in Fig. 3

A nontrivial test for the quality accuracy of our calculation is the numerical verification of the optical theorem Eq. (3.23). Our results are given for selected energies in Table Here we show two sets of cross sections, distinguished by the superscripts $q_{0}$ and $q$ for the total and elastic cross section. The superscripts indicate that the calculation is carried out by choosing the z-axis either parallel to $\hat{\mathbf{q}}_{\mathbf{0}}$ or to $\hat{\mathbf{q}}$. Performing the calculation with two different choices of the z-axis is a nontrivial test for our choice of independent variables as well as for the entire calculation. The total break-up cross section is also calculated in a coordinate system in which the z-axis is parallel to $\hat{\mathbf{p}}$, indicated by $\sigma_{b r}^{p}$. The calculations are based on the largest grids given in Table \and show a very good agreement of the results obtained in the different coordinate systems. This indicates the numerical rotational invariance of our calculations.

On top of convergence tests for the Padé summation, we insert the resulting amplitude $\hat{T}$ again into the integral of Eq. (2.19), leading to a second amplitude $\hat{T}^{\prime}$. Both amplitudes should be identical within our numerical errors. This is checked by evaluating the cross sections again using the second amplitude. We document the results in Table III for the differential elastic cross section at selected angles. The table shows excellent agreement of the two values of the cross section

Finally, another highly nontrivial test of our calculation is the independence of the cross sections from the arbitrary angle $\varphi_{q_{0}}$ and the sign of $\sin \left(\varphi_{p}-\varphi_{q_{0}}\right)$. This is documented in Tables IV and $\nabla$ for the energy $E=3 \mathrm{MeV}$. In order the check the rotational invariance numerically, the calculations are carried out in two different coordinate systems, one where $\mathbf{q}_{0}$ is parallel the $z$-axis, and one where $\mathbf{q}$ is parallel to $z$. Both tables show excellent agreement for the cross sections, and thus we conclude that our choice of variables is correct.

\section{SCATTERING CALCULATIONS AT INTERMEDIATE ENERGIES}

Through we neglect spin and iso-spin degrees of freedom and stay in a strictly nonrelativistic framework, we nevertheless can provide first qualitative insights for various cross sections in three-body scattering in the intermediate energy regime which we define from $200 \mathrm{MeV}$ to $1 \mathrm{GeV}$ projectile energy. The focus of our investigations will be the question which orders of rescattering in the two-body $t$-matrix are needed to come close to the exact result, namely the solution of the Faddeev equation.

As a model two-body interaction we choose a superposition of two Yukawa interactions of Malfliet-Tjon type [25]

$$
V\left(\mathbf{p}^{\prime}, \mathbf{p}\right)=\frac{1}{2 \pi^{2}}\left(\frac{V_{R}}{\left(\mathbf{p}^{\prime}-\mathbf{p}\right)^{2}+\mu_{R}^{2}}-\frac{V_{A}}{\left(\mathbf{p}^{\prime}-\mathbf{p}\right)^{2}+\mu_{A}^{2}}\right) .
$$

The parameters are given in Table VI and fitted such that the potential supports a two-body bound state, the 
'deuteron', at $-2.23 \mathrm{MeV}$. As first result we show in Fig. 4 the total cross section together with the total elastic and total break-up cross section as function of the laboratory projectile energy. In addition, the total cross section is also evaluated via the optical theorem as a test of our numerics. This duplicates the information already given in Table III. We see that the optical theorem is quite well fulfilled. The figure shows that at roughly 1 GeV the total elastic and total break-up cross section become equal in magnitude in our model.

Next we show in Fig. 4 the angular distribution in elastic scattering for a set of selected energies. In addition to the exact Faddeev result, the cross sections are evaluated in first order in the two-body $t$-matrix, second order in $t$, third order in $t$, and 4 th order in $t$, and displayed. First we notice that with increasing energy the cross section in forward direction decreases. Furthermore, for all energies shown, the first rescattering (2nd order in $t$ ) always increases the cross section, and subsequent rescatterings lower it again. As expected, for the lowest energy, $0.2 \mathrm{GeV}$, rescattering terms of higher order are important, and even the 4th order is not yet close to the full result. The same is true for $0.8 \mathrm{GeV}$. We notice, that even at $1 \mathrm{GeV}$ two rescattering terms (3rd order in $t$ ) are necessary to come into the vicinity of the final result. The same is true for $0.5 \mathrm{GeV}$.

In view of the standard ' $t$ - $\rho$ ' impulse approximation for the optical potential in nucleon-nucleus scattering employed at intermediate energies 26, it is interesting to notice that the first order result in $t$ in our model study is quite insufficient. Even at energies larger than $0.5 \mathrm{GeV}$ rescattering corrections up to the 3rd order are required to come close to the exact result for small scattering angles. Therefore it seems to be likely that the first order impulse approximation in nucleon-nucleus scattering is insufficient.

In case of inelastic processes we first regard the semi-exclusive reaction $\mathrm{d}\left(\mathrm{N}, \mathrm{N}^{\prime}\right)$ where only one nucleon is detected. We choose three different laboratory energies, $200 \mathrm{MeV}, 500 \mathrm{MeV}$, and $1 \mathrm{GeV}$ and show the inclusive cross section as a few selected angles for the detected nucleon. The results are shown in Figs. 6] through [10]

At $0.2 \mathrm{GeV}$ the semi-exclusive cross section is given in Fig. 6 for the emission angle $24^{\circ}$ and in Fig. 7 for the emission angle $39^{\circ}$. Both figures show in the upper panel the entire energy range of the emitted particle. Since the cross section varies by two orders of magnitude, we display it in a logarithmic scale. In order to better flash out the peak structures, the two lower panels show the high and low energies of the emitted particle in a linear scale. Together with the full solution of the Faddeev equation (solid line) we display the sums of the lowest orders of the multiple scattering series as indicated in the figure. The peak at the highest energy of the emitted particle is the socalled final state interaction (FSI) peak, which only develops when rescattering terms are taken into account. This peak is a general feature of semi-exclusive scattering and is present for all energies. The next peak is the quasi-free (QFS) peak, and one sees that at both angles one needs at least rescattering of 4 th order to come close to the full result. However, in contrast to the smaller angle, at the larger angle, $39^{\circ}$ in Fig. [7 the first order result for the larger energies is surprisingly close to the full solution, though the multiple scattering series is by no means converged, as the following higher orders indicate. We also observe that the QFS peak moves to lower energies of the emitted particle with increasing emission angle. At both angles the very low energies of the emitted particle exhibit a strong peak in first order, which is considerably lowered by the first rescattering. Here the calculation up to 3rd order in the multiple scattering series seems already sufficient.

For $0.5 \mathrm{GeV}$ incident energy the semi-exclusive cross section is given in Fig. 8 for the emission angle $24^{\circ}$ and in Fig. 9] for the emission angle $36^{\circ}$. We again see three peaks along along the energy axis of the detected nucleon, the FSI and QFS peaks as well as the peak at the extreme low energy of the emitted particle. Again we see that the results based on first and second order in $t$ alone are quite insufficient and higher order rescatterings can not be neglected. It is also interesting to observe that at $24^{\circ}$, Fig. 8 , the third and higher order rescattering terms shift the peak to higher energies, whereas at the larger angle of $36^{\circ}$ the peak positions of the various orders coincide more or less and agree with the peak position of the full calculation. Again, for the peak for the very low energies of the emitted particle, the 3rd order calculation agrees already quite well with the full result.

At $1 \mathrm{GeV}$ the situation is similar. As examples we have selected two angles, $18^{\circ}$ displayed in Fig. 10 and $30^{\circ}$ displayed in Fig. 11] For the small emission angle the second rescattering shifts the QFS peak towards higher energies, at the larger angle this is not the case. Our studies indicate that this is a general phenomemon occurring at all energies under consideration. There exists a critical maximum energy $E_{1}^{\max }$ of the emitted particle, corresponding to a specific emission angle, at which such a shift in the QFS peak through higher order rescattering terms can occur. At $0.5 \mathrm{GeV}$ projectile energy this maximum energy is $0.44 \mathrm{GeV}$, at $1 \mathrm{GeV}$ it is $0.88 \mathrm{GeV}$. If one considers the ratio $\frac{E_{1}^{\max }}{E_{l a b}}$, then one finds for both cases that if this ratio is larger than 0.8, the QSF peak is shifted by higher order rescattering terms. This could be interpreted as an interference between the QFS and the FSI mechanisms. If this ratio is smaller than 0.8 , then the FSI peak is small and higher orders in the multiple scattering series do not change the position of the QFS peak. In addition, it seems that at the larger angle (Fig. 11) the multiple scattering series converges a little faster with respect to the higher orders compared to the smaller angle (Fig. 10). The final result for the peak at the very low energy of the emitted particle is as before reached with two rescattering contributions. It is remarkable that for the energies between about 200 and $500 \mathrm{MeV}$ of the emitted particle the first rescattering contributes almost an order of magnitude to the cross section. 
We can make a first contact to calculations based on realistic nucleon-nucleon (NN) forces. In Ref. [17] the semiexclusive process $d(p, n)$ has been determined in first order in $t$ based on the NN potentials AV18 27] and Bonn-B [28. In the upper panel of Fig. 12 we compare our first order calculation at projectile laboratory energy $495 \mathrm{MeV}$ and $18^{\circ}$ emission angle with the first order calculations from Ref. [17] based on the two realistic potentials. The position of the peak is only determined through kinematics, thus the peak position coincides for all three calculations. Though our model calculation refers to bosons and the potential contains only the crude features of a central short range repulsion and intermediate range attraction, the magnitudes of the cross sections differ only by roughly $20 \%$. In the lower panel we show the contributions of the first orders of the multiple scattering series successively summed together with the exact solution of the Faddeev equation for our model. At this angle and energy the contribution of the first rescattering (2nd-order in the multiple scattering series) is quite weak, the contributions of the next two orders are large and lower the size of the peak. At the very high energies of the emitted particle, the 4 th order in the multiple scattering series is still not yet close to the exact result. Therefore we conjecture that at this energy calculations with realistic forces will also require higher order rescattering contributions.

Finally we comment on a recently measured and analyzed reaction $p d \rightarrow(p p) n$ at high momentum transfer [29, 30]. In this experiment the break-up configuration has been chosen such that the neutron is ejected at extreme backward angles, and the two protons at extreme forward angles. The measurement was carried out at GeV laboratory energies. The experimental data [29] have been analyzed in Ref. [30] using first and second order processes in the NN $t$-matrix including a $\Delta$-isobar mechanism. Within our nonrelativistic toy model for three bosons we are of course unable to analyze the data. However, within our model we can give a clear answer whether higher order rescattering processes are essential in this reaction. In [29, 30] the data are integrated over a small interval of the relative $p p$ energy between 0 and $3 \mathrm{MeV}$, and averaged over the neutron c.m. angle in the interval between $172^{\circ}$ and $180^{\circ}$. In our qualitative study we fix the c.m. angle of one particle at $180^{\circ}$, but integrate over the relative energy $E_{p p}=p^{2} / m$ of the two other particles between 0 and $3 \mathrm{MeV}$. Thus, we evaluate the cross section

$$
\frac{d \sigma}{d \Omega_{q}}=(2 \pi)^{4}\left(\frac{2}{3}\right)^{2} \frac{m^{2}}{q_{0}} \int_{0}^{\sqrt{m E_{p p}}} d p p^{2} q \int d \hat{p} \mid U_{0}\left(p, x_{p}, x_{p q}^{q_{0}}, x_{q}=-1, q,\left.q_{0}\right|^{2}\right.
$$

Since we choose the z-axis parallel to $\hat{\mathbf{q}}_{\mathbf{0}}$, and $\hat{\mathbf{q}}$ is antiparallel to $\hat{\mathbf{q}}_{\mathbf{o}}$, the $\varphi_{p}$ dependence is directly given by $x_{p q}^{q_{0}}=\cos \varphi_{p}$. Our calculations are carried out for projectile laboratory energies between 0.2 and $1 \mathrm{GeV}$, and are displayed in Fig. 13 . Here we compare different low orders in the two-body $t$-matrix with the full solution of the Faddeev equation. We notice that all our calculations exhibit a smooth fall-off as function of the projectile energy. This behavior is present in the data of Ref. 29|. None of our calculations shows a dip structure around $0.7 \mathrm{GeV}$ as indicated for some of the calculations in Ref. 30]. The reason may be that our calculation is carried out in three dimension, i.e. all partial waves are included exactly, where as in Ref. 30 only the lowest partial waves are considered. At low projectile energies rescattering terms of higher order still give considerable contributions to the cross section. At 1 GeV the first order calculation is an order of magnitude smaller than the result of the full calculation. It is interesting to notice that at $1 \mathrm{GeV}$ the contribution from the first rescattering is relatively small,and one needs to go to the 3rd order in $t$ to come close to the full result for this particular break-up configuration.

In addition to the specific break-up configuration described above, a measurement of the extreme backward scattering elastic $p d$ cross section has been investigated in [30. Instead of a forward scattered $p p$ pair with very small relative energy, one now has a forward going deuteron. This situation corresponds to elastic scattering from a deuteron at backward angle. To investigate the influence of rescattering for this reaction we plot in Fig. 14 the backward angle of the elastic cross section at energies from 0.2 to $1 \mathrm{GeV}$, and compare the result of the full Faddeev calculation with calculations based on low orders in the multiple scattering series. The figure shows that the first order calculation is insufficient over the entire energy regime considered, except of course for the crossing point around $0.5 \mathrm{GeV}$. The first rescattering contribution (2nd order calculation), though close at $0.2 \mathrm{GeV}$, is insufficient below roughly $0.9 \mathrm{GeV}$. The figure also shows that at about $0.9 \mathrm{GeV}$ the relative magnitude of contributions from the second and higher rescattering become small. Thus, we conclude that at $1 \mathrm{GeV}$ one needs at least one rescattering to be in the vicinity of the full result for the elastic cross section at the backward angle.

\section{SUMMARY AND CONCLUSIONS}

In this study we perform fully converged Faddeev calculations for three identical bosons interacting by non-separable forces in the intermediate energy range between about 0.2 and $1.0 \mathrm{GeV}$. To the best of our knowledge these are the first calculations of this kind. The key point is to neglect the partial wave decomposition generally used at low energies and to work directly with momentum vectors. Thus all partial waves are exactly included. Important is the suitable choice of variables. Besides the two magnitudes of the two relative Jacobi momenta $\mathbf{p}$ and $\mathbf{q}$ we choose the angles 
between the vectors $\mathbf{p}$ and $\mathbf{q}_{\mathbf{0}}$ and between $\mathbf{q}$ and $\mathbf{q}_{\mathbf{0}}$, where $\mathbf{q}_{\mathbf{0}}$ is the projectile momentum. The fifth variable is the angle between the two planes spanned by $\mathbf{p}, \mathbf{q}_{\mathbf{0}}$ and $\mathbf{q}, \mathbf{q}_{\mathbf{0}}$. In the technical piece of the work we introduce for the first time a spline based integration of the moving logarithmic singularities, which is a very valuable alternative to procedures used so far. The numerical results are converging as documented in Section III. In Section V we show elastic and inelastic (break-up) cross sections in the above mentioned intermediate energy range. We focus on the question how many orders of rescattering beyond the often used first order calculation in the two-body $t$-matrix are needed to come close to the full Faddeev result. We find that in nearly all cases studied processes of at least 2 nd and 3rd order rescattering are required. Whether this will be also required in performing calculations with realistic dynamical inputs has to be seen in the future.

In one case we can make first contact to a result based on the NN forces AV18 and Bonn B, which are considered to be realistic in the sense that they describe all NN data below $350 \mathrm{MeV}$ extremely well. This was the semi-exclusive cross section at $495 \mathrm{MeV}$ evaluated in first order in the NN t-matrix. Of course, at that energy AV18 and Bonn-B are at the upper limit of their applicability. Despite our simple two-body model force, a superposition of two Yukawa interactions, one attractive the other repulsive, our results turns out to be within about $20 \%$ to the calculation based on the realistic models. This shows that our investigations might allow some conclusions about results based on present and future models with more dynamical inputs.

As a first example for considering data in the light of our toy model we study the extreme backward elastic dN scattering over the energy range from 0.2 to $1.0 \mathrm{GeV}$. We find that first order results in the two-body $t$-matrix are totally insufficient and only around $1 \mathrm{GeV}$ the first order rescattering comes close to the full result. Parallel to those data in elastic scattering in 29, 30 the complete break up process d(p,n)pp has also been investigated. Here the neutron was ejected antiparallel to the beam direction and the two protons at extreme forward angles with a very small relative energy. Again we study the significance of rescattering processes, and find that for this particular break-up configuration two rescatterings are necessary to get close to the result of the full Faddeev calculation.

In conclusion we can say that the three-body Faddeev equations can be safely solved at intermediate energies using directly momentum vectors. Calculations based on partial wave decomposition would be hardly feasible at these energies.

Further studies scanning the complete three-body phase space for the total break-up are underway. This may be important in order to shed light on previous theoretical analysis of $\mathrm{p}(\mathrm{d}, \mathrm{ppn})$ reactions which relied on low order reaction mechanisms.

Based on our current experience it appears that if low order rescattering processes will turn out to be sufficient for certain phase space regions, realistic calculations including spin and isospin will be feasible, even including threebody forces. A first step evaluating the $\mathrm{d}(\mathrm{p}, \mathrm{n}) \mathrm{pp}$ break-up cross section in first order with a currently used two-pion exchange three-nucleon force model is already under way [31]. What is badly needed now are realistic models for nuclear forces in the intermediate energy regime we study. This paper allows us to conclude that it will be feasible to extend the calculations to realistic dynamics.

In Ref. [17] the effect of relativistic kinematics was studied in the (p,n) charge exchange reaction on deuterium between 0.1 and $0.5 \mathrm{GeV}$ in a first order Faddeev calculation. This work concluded that the effects due to relativistic kinematics are quite visible at $0.5 \mathrm{GeV}$, specifically in the location of the position of the QFS peak, which is purely determined by kinematics. Therefore, we should expect that the relativistic kinematics will influence our results, especially at energies larger than $0.5 \mathrm{GeV}$. Of course, there are other dynamical relativistic effects. For energies below $\sim 0.25 \mathrm{GeV}$ those relativistic effects were studied in neutron-deuteron elastic scattering in Ref. 32. There it was found that the combination of relativistic effects consistently incorporated is negligible below $0.1 \mathrm{GeV}$, and manifests itself at $0.25 \mathrm{GeV}$ mostly at large scattering angles. What happens at the energies we considered when relativistic effects (kinematical and dynamical ones) are incorporated is uncharted territory so far, and we want to refrain from speculation. Work in this direction is however underway.

\section{Acknowledgments}

This work was performed in part under the auspices of the U. S. Department of Energy under contract No. DEFG02-93ER40756 with Ohio University. We thank the National Energy Research Supercomputer Center (NERSC) for the use of their facilities. We thank I. Fachruddin for allowing us to use his results for comparison with ours. One of us (W.G.) would like to thank K. Miyagawa for fruitful collaboration on the spline based integration of the logarithmic singularities. 


\section{APPENDIX A: THE $\varphi^{\prime \prime}$ INTEGRATION}

According to Eqs. (2.19), 2.18), and 2.20) the $\varphi^{\prime \prime}$-integration for fixed $p, q, x_{p}, x_{q}, x_{p q}^{q_{0}}, q^{\prime \prime}$, and $x^{\prime \prime}$ can be written as

$$
I\left(\varphi_{q_{0}}, \varphi_{p}\right)=\int_{0}^{2 \pi} F\left[\cos \left(\varphi^{\prime \prime}-\varphi_{q_{0}}\right)\right] G\left[\cos \left(\varphi^{\prime \prime}-\varphi_{p}\right)\right] d \varphi^{\prime \prime}
$$

where the $F$ and $G$ are known functions from $\hat{t}_{s}$ and $\hat{T}$. The substitution $\varphi^{\prime}=\varphi^{\prime \prime}-\varphi_{q_{0}}$ leads to

$$
\begin{aligned}
I\left(\varphi_{q_{0}}, \varphi_{p}\right) & =\int_{0}^{2 \pi} F\left[\cos \varphi^{\prime}\right] G\left[\cos \left(\varphi^{\prime}-\left(\varphi_{p}-\varphi_{q_{0}}\right)\right)\right] d \varphi^{\prime} \\
& \equiv I\left(\varphi_{q_{0}}-\varphi_{p}\right) .
\end{aligned}
$$

Moreover, splitting this integral as

$$
\begin{aligned}
I\left(\varphi_{q_{0}}-\varphi_{p}\right) & =\int_{0}^{\pi} F\left[\cos \varphi^{\prime}\right] G\left[\cos \left(\varphi^{\prime}-\left(\varphi_{p}-\varphi_{q_{0}}\right)\right)\right] d \varphi^{\prime} \\
& +\int_{\pi}^{2 \pi} F\left[\cos \varphi^{\prime}\right] G\left[\cos \left(\varphi^{\prime}-\left(\varphi_{p}-\varphi_{q_{0}}\right)\right)\right] d \varphi^{\prime}
\end{aligned}
$$

and substituting $\varphi^{\prime}=2 \pi-\varphi^{\prime \prime}$ in the second integral, one obtains

$$
I\left(\varphi_{p}-\varphi_{q_{0}}\right)=\int_{0}^{\pi} F\left[\cos \varphi^{\prime \prime}\right]\left(G\left[\cos \left(\varphi^{\prime \prime}-\left(\varphi_{p}-\varphi_{q_{0}}\right)\right)\right]+G\left[\cos \left(\varphi^{\prime \prime}+\left(\varphi_{p}-\varphi_{q_{0}}\right)\right)\right]\right) d \varphi^{\prime \prime} \equiv I\left(\left|\varphi_{p}-\varphi_{q_{0}}\right|\right) .
$$

Consequently, the result for the $\varphi$-integration in Eq. A2 or Eq. A4 does not depend on the choice of the sign in $\sin \left(\varphi_{p}-\varphi_{q_{0}}\right)= \pm \sqrt{1-\cos ^{2}\left(\varphi_{p}-\varphi_{q_{0}}\right)}$. Only $\cos \left(\varphi_{p}-\varphi_{q_{0}}\right)$ is fixed by Eq. (2.21), and has to be known.

Since the integral $I$ in Eq. (A4) depends only on the difference of the angles $\left(\varphi_{p}-\varphi_{q_{0}}\right)$, one can choose $\varphi_{q_{0}}$ arbitrarily, and thus $\sin \varphi_{q_{0}}$ and $\cos \varphi_{q_{0}}$ required in Eq. 2.18). Moreover, the trivial identities

$$
\begin{aligned}
\cos \varphi_{p} & =\cos \varphi_{q_{0}} \cos \left(\varphi_{p}-\varphi_{q_{0}}\right)-\sin \varphi_{q_{0}} \sin \left(\varphi_{p}-\varphi_{q_{0}}\right) \\
\sin \varphi_{p} & =\sin \varphi_{q_{0}} \cos \left(\varphi_{p}-\varphi_{q_{0}}\right)+\cos \varphi_{q_{0}} \sin \left(\varphi_{p}-\varphi_{q_{0}}\right)
\end{aligned}
$$

are the input for $\cos \left(\varphi_{p}-\varphi^{\prime \prime}\right)$ needed in Eq. (2.20). The arbitrary choice of $\varphi_{q_{0}}$ is a good check for the numerical correctness of the choice of variables, and we carried out those tests.

[1] J.L. Friar, G.L. Payne, W. Glöckle, D. Hüber, H. Witala, Phys. Rev. C 51, 2356 (1995).

[2] H. Witala, Th. Cornelius, W. Glöckle, Few-Body Systems 3, 123 (1988).

[3] A. Kievsky, M. Viviani, and S. Rosati, Phys. Rev. C 64, 024002 (2001).

[4] M. Viviani, A. Kievsky, and S. Rosati, Few-Body Systems 30, 39 (2001).

[5] W. Glöckle, et. al., Phys. Rep. 274, 107 (1996).

[6] W. Glöckle in 'Scattering, Scattering and Inverse Scattering in Pure and Applied Science', Eds. R. Pike and P. Sabatier, Academic Press, p. 1339-1359 (2002).

[7] J. Kuros-Zolnericzuk, et al., Phys. Rev. C 66, 024004 (2002).

[8] H. Witala, et al., Phys. Rev. C 63, 024007 (2001).

[9] K. Chmielewski, A. Deltuva, A.C. Fonseca, S. Nemoto, P.U. Sauer, Phys. Rev. C 67, 014002 (2003).

[10] A. Arriaga, V. R. Pandharipande, and R. B. Wiringa, Phys. Rev. C 52, 2362 (1995).

[11] J. Carlson, Phys. Rev. C 36, 2026 (1987).

[12] J. Carlson, Phys. Rev. C 38, 1879 (1988).

[13] J. G. Zabolitzki, K. E. Schmidt, and M. H. Kalos, Phys. Rev. C 25, 1111 (1982).

[14] J. Carlson and R. Schiavilla, Rev. Mod. Phys. 70, 743 (1998).

[15] Ch. Elster, W. Schadow, A. Nogga and W. Glöckle, Few-Body System 27, 83 (1999).

[16] H. Liu, Ch. Elster and W. Glöckle, Few-Body System 33, 241 (2003).

[17] I. Fachruddin, Ch. Elster, W. Glöckle, Phys. Rev. C68, 054003 (2003).

[18] W. Glöckle, The Quantum Mechanical Few-Body Problem (Springer-Verlag, Berlin-Heidelberg, 1983).

[19] W. Schadow, Ch. Elster, W. Glöckle, Few-Body Systems 28, 15 (2000). 
[20] Ch. Elster, J. H. Thomas and W. Glöckle, Few-Body System 24, 55 (1998).

[21] G. A. Baker and J. L. Gammel, ed., The Padé Approximation in Theoretical Physics (Academic Press, New York, 1970).

[22] D. Hüber, Ph.D. thesis, Bochum University, Bochum, Germany, 1996.

[23] D. Hüber, H. Witala, A. Nogga, W. Glöckle and H. Kamada, Few-Body System, 22, 107 (1997).

[24] H. Liu, PhD thesis, Ohio University, in preparation.

[25] R.A. Malfliet, A.J. Tjon, Nucl. Phys. A, 127, 161 (1969).

[26] L. Ray, G.W. Hoffmann, Phys. Rev. C31, 538 (1985); R. Crespo, R.C. Johnson, J.A. Tostevin, Phys. Rev. C44, R1735 (1991); Ch. Elster, T. Cheon, E.F. Redish, P.C. Tandy, Phys. Rev. C41, 814 (1990).

[27] R. B. Wiringa, V. G. J. Stoks, R. Schiavilla, Phys. Rev. C51, 38 (1995).

[28] R. Machleidt, Adv. Nucl. Phys. 19, 189 (1989).

[29] V. Komarov et al., Phys. Lett. B 553, 179 (2003).

[30] J. Haidenbauer, Y.N. Uzikov, Phys. Lett. B 562, 227 (2003).

[31] I. Fachruddin, private communication.

[32] H. Witala, J. Golak, W. Glöckle, H. Kamada, Phys. Rev. C71 054001 (2005). 


\begin{tabular}{|c|ccccccc|ccc|c|}
\hline$E_{l a b}[\mathrm{GeV}]$ & $p$ & $x_{p}$ & $x_{p q}^{q_{0}}$ & $x_{q}$ & $q, q^{\prime \prime}$ & $x^{\prime \prime}$ & $\varphi^{\prime \prime}$ & $\sigma_{o p t}[\mathrm{mb}]$ & $\sigma_{e l}[\mathrm{mb}]$ & $\sigma_{b r}[\mathrm{mb}]$ & $\sigma_{e l}+\sigma_{b r}[\mathrm{mb}]$ \\
\hline 0.01 & 49 & 4 & 4 & 4 & 49 & 4 & 4 & 1913.48 & 1799.08 & 67.81 & 1866.89 \\
& 49 & 8 & 8 & 8 & 49 & 8 & 8 & 1886.84 & 1807.50 & 70.14 & 1877.64 \\
& 49 & 12 & 12 & 12 & 49 & 12 & 12 & 1904.99 & 1820.77 & 73.75 & 1894.52 \\
& 49 & 16 & 16 & 16 & 49 & 16 & 16 & 1903.22 & 1820.46 & 73.20 & 1893.66 \\
\hline \hline 0.1 & 49 & 12 & 12 & 12 & 49 & 12 & 12 & 335.57 & 259.95 & 83.10 & 343.05 \\
& 49 & 16 & 16 & 16 & 49 & 16 & 16 & 343.17 & 265.83 & 75.84 & 341.67 \\
& 49 & 23 & 23 & 16 & 49 & 16 & 20 & 344.34 & 270.05 & 76.23 & 346.28 \\
& 49 & 23 & 23 & 24 & 49 & 24 & 20 & 346.16 & 272.04 & 76.55 & 348.59 \\
\hline \hline \multirow{2}{*}{0.5} & 49 & 12 & 12 & 12 & 49 & 12 & 12 & 40.17 & 12.05 & 66.32 & 78.37 \\
& 49 & 16 & 16 & 16 & 49 & 16 & 16 & 65.62 & 47.76 & 32.73 & 80.49 \\
& 49 & 20 & 20 & 16 & 49 & 16 & 20 & 65.93 & 47.61 & 38.16 & 86.22 \\
& 49 & 20 & 16 & 20 & 49 & 20 & 16 & 85.19 & 61.16 & 28.84 & 90.00 \\
\hline & 49 & 20 & 20 & 20 & 49 & 20 & 20 & 85.71 & 61.30 & 29.86 & 91.19 \\
& 49 & 24 & 20 & 20 & 49 & 20 & 20 & 85.72 & 61.24 & 30.56 & 91.80 \\
& 49 & 20 & 20 & 24 & 49 & 24 & 20 & 102.17 & 64.96 & 33.74 & 98.70 \\
& 49 & 23 & 23 & 24 & 49 & 24 & 20 & 110.35 & 64.28 & 36.42 & 100.70 \\
\hline \hline
\end{tabular}

TABLE I: The total elastic and break-up cross sections together with the total cross section extracted via the optical theorem calculated from a Malfliet-Tjon type potential at two selected energies $(0.01$ and $0.5 \mathrm{GeV})$ as function of the grid points. The double prime quantities are the integration variables. The calculations are carried out in the coordinate system in which $q_{0}$ is aligned parallel to the $\mathrm{z}$-axis.

\begin{tabular}{|c|cc|ccc|cc|}
\hline$E_{l a b}[\mathrm{GeV}]$ & $\sigma_{e l}^{q_{0}}[\mathrm{mb}]$ & $\sigma_{e l}^{q}[\mathrm{mb}]$ & $\sigma_{b r}^{q_{0}}[\mathrm{mb}]$ & $\sigma_{b r}^{q}[\mathrm{mb}]$ & $\sigma_{b r}^{p}[\mathrm{mb}]$ & $\sigma_{o p t}^{q_{0}}[\mathrm{mb}]$ & $\sigma_{o p t}^{q}[\mathrm{mb}]$ \\
\hline 0.003 & 2561.74 & 2561.14 & 0.0 & 0.0 & 0.0 & 2562.65 & 2562.65 \\
\hline 0.01 & 1820.46 & 1820.51 & 73.20 & 73.55 & 73.13 & 1903.22 & 1902.56 \\
\hline 0.1 & 272.04 & 272.20 & 76.55 & 75.18 & 75.08 & 346.16 & 346.16 \\
\hline 0.5 & 64.28 & 64.61 & 36.42 & 36.39 & 35.55 & 110.35 & 110.35 \\
\hline 1.0 & 21.90 & 21.90 & 23.44 & 23.46 & 23.40 & 49.59 & 49.59 \\
\hline
\end{tabular}

TABLE II: The total elastic cross section, total breakup cross section and total cross section extracted via the optical theorem calculated in different coordinate systems at selected energies. The choice of coordinate system, i.e. which vector is aligned parallel to the z-axis, is indicated by the superscripts $q_{0}, q$ and $p$. 


\begin{tabular}{|c|c|c|c|}
\hline$E_{l a b}[\mathrm{GeV}]$ & $\theta_{c m}[\mathrm{deg}]$ & $\left.\frac{d \sigma^{e l}}{d \Omega_{c m}}\right|_{T}[\mathrm{mb}]$ & $\left.\frac{d \sigma_{c m}^{e l}}{d \Omega_{c m}}\right|_{T^{\prime}}[\mathrm{mb}]$ \\
\hline 0.01 & 0.0 & 537.536 & 537.536 \\
& 21.8 & 420.036 & 420.036 \\
& 62.1 & 70.726 & 70.725 \\
& 93.4 & 38.289 & 38.289 \\
& 151.5 & 227.899 & 227.899 \\
\hline 0.2 & 0.0 & 676.821 & 676.821 \\
& 21.8 & 148.880 & 148.880 \\
& 62.1 & 0.363 & 0.363 \\
& 93.4 & 0.223 & 0.223 \\
& 151.5 & 0.010 & 0.010 \\
\hline 0.5 & 0.0 & 519.389 & 519.389 \\
& 21.8 & 16.209 & 16.209 \\
& 26.3 & 4.430 & 4.430 \\
& 62.1 & 0.088 & 0.088 \\
& 93.4 & 0.005 & 0.005 \\
& 151.5 & 0.004 & 0.004 \\
\hline \multirow{2}{*}{1.0} & 0.0 & $3.903 \times 10^{+2}$ & $3.903 \times 10^{+2}$ \\
& 21.8 & $5.325 \times 10^{-1}$ & $5.325 \times 10^{-1}$ \\
& 62.1 & $4.072 \times 10^{-4}$ & $4.072 \times 10^{-4}$ \\
& 93.4 & $2.678 \times 10^{-3}$ & $2.678 \times 10^{-3}$ \\
& 151.5 & $3.705 \times 10^{-4}$ & $3.703 \times 10^{-4}$ \\
\hline
\end{tabular}

TABLE III: The elastic differential cross sections at different energies for selected scattering angles. The cross section labeled by $T$ results from the converged solution of the integral equation Eq. (2.19). The column labeled $T^{\prime}$ is calculated by reinserting the original solution into the Faddeev equation with $T^{\prime}=t P+t G_{0} P T$. The calculations are based on a Malfliet-Tjon type potential as described in the text.

\begin{tabular}{|c|cc|cc|}
\hline$\varphi_{q_{0}}[\mathrm{rad}]$ & $\sigma_{e l}^{q_{0}}[\mathrm{mb}]$ & $\sigma_{e l}^{q}[\mathrm{mb}]$ & $\sigma_{o p t}^{q_{0}}[\mathrm{mb}]$ & $\sigma_{o p t}^{q}[\mathrm{mb}]$ \\
\hline 0.0 & 2561.736 & 2561.138 & 2562.649 & 2562.649 \\
\hline$\frac{\pi}{2}$ & 2560.885 & 2560.729 & 2562.496 & 2562.496 \\
\hline$\pi$ & 2561.550 & 2561.206 & 2562.091 & 2562.091 \\
\hline
\end{tabular}

TABLE IV: The total elastic cross sections at $E_{l a b}=3.0 \mathrm{MeV}$ calculated for different values of the angle $\varphi_{q_{0}}$ with the + sign of $\sin \left(\varphi_{p}-\varphi_{q_{0}}\right)$. The calculations are carried out in two different coordinate systems, characterized by the superscripts $q_{0}$ and $q$, which indicate, which vector is chosen to be parallel to the z-axis.

\begin{tabular}{|c|cc|cc|}
\hline $\operatorname{sign}\left[\sin \left(\varphi_{p}-\varphi_{q_{0}}\right)\right]$ & $\sigma_{e l}^{q_{0}}[\mathrm{mb}]$ & $\sigma_{e l}^{q}[\mathrm{mb}]$ & $\sigma_{o p t}^{q_{0}}[\mathrm{mb}]$ & $\sigma_{o p t}^{q}[\mathrm{mb}]$ \\
\hline+ & 2561.736 & 2561.138 & 2562.649 & 2562.649 \\
\hline- & 2559.674 & 2559.536 & 2560.091 & 2560.091 \\
\hline
\end{tabular}

TABLE V: The total elastic cross sections at $E_{l a b}=3.0 \mathrm{MeV}$ calculated for different $\operatorname{sign}$ of $\sin \left(\varphi_{p}-\varphi_{q_{0}}\right)$ where $\varphi_{q_{0}}=0$. The meaning of the superscripts is the same as in Table IV

\begin{tabular}{|c|c|c|c|c|}
\hline$V_{A}[\mathrm{MeV} \mathrm{fm}]$ & $\mu_{A}\left[\mathrm{fm}^{-1}\right]$ & $V_{R}[\mathrm{MeV} \mathrm{fm}]$ & $\mu_{R}\left[\mathrm{fm}^{-1}\right]$ & $E_{d}[\mathrm{MeV}]$ \\
\hline-626.8932 & 1.550 & 1438.7228 & 3.11 & -2.2307 \\
\hline
\end{tabular}

TABLE VI: The parameters and deuteron binding energy for the Malfliet-Tjon type potential of our calculation. As conversion factor We use units such that $\hbar c=197.3286 \mathrm{MeV} \mathrm{fm}=1$. 


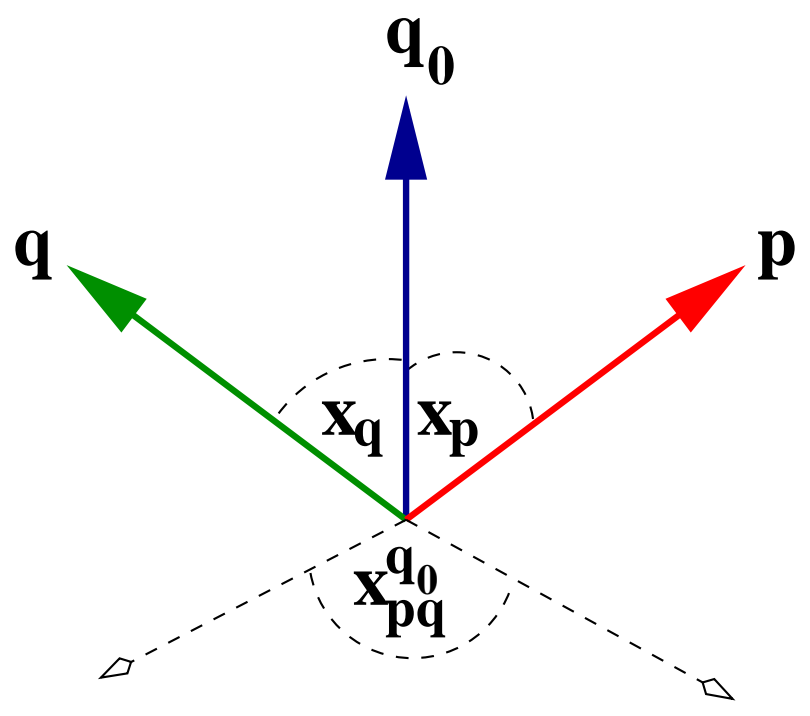

FIG. 1: The geometry of three vectors $\mathbf{q}_{\mathbf{0}}, \mathbf{q}$, and $\mathbf{p}$ relevant in the three-body scattering problem. The independent angle variables $x_{q}, x_{p}$, and $x_{p q}^{q_{0}}$ as defined in Section II are indicated. The dashed arrows represent the normal vectors $\left(\mathbf{q}_{\mathbf{0}} \times \mathbf{q}\right)$ and $\left(\mathbf{p} \times \mathbf{q}_{\mathbf{0}}\right)$.

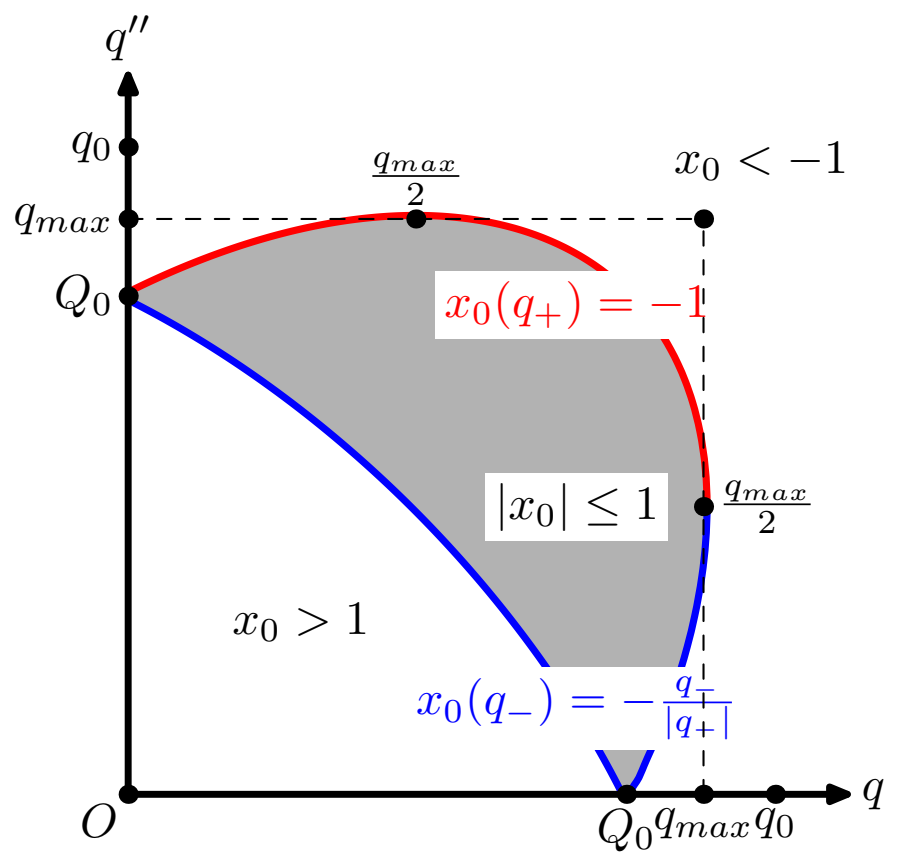

FIG. 2: The region of singularities of the free three-particle propagator as function of the momenta $q$ and $q^{\prime \prime}$. The shaded area in the $q-q^{\prime \prime}$ plane indicates the region where $\left|x_{0}\right| \leq 1$, i.e. the region where a pole in the $x^{\prime \prime}$-integration occurs. This region is is enclosed by the bounding curves $q_{+}$and $q_{-}$, which contain the logarithmic singularity as function of $q^{\prime \prime}$ as given in Eqs. 4.5 and 4.6. 

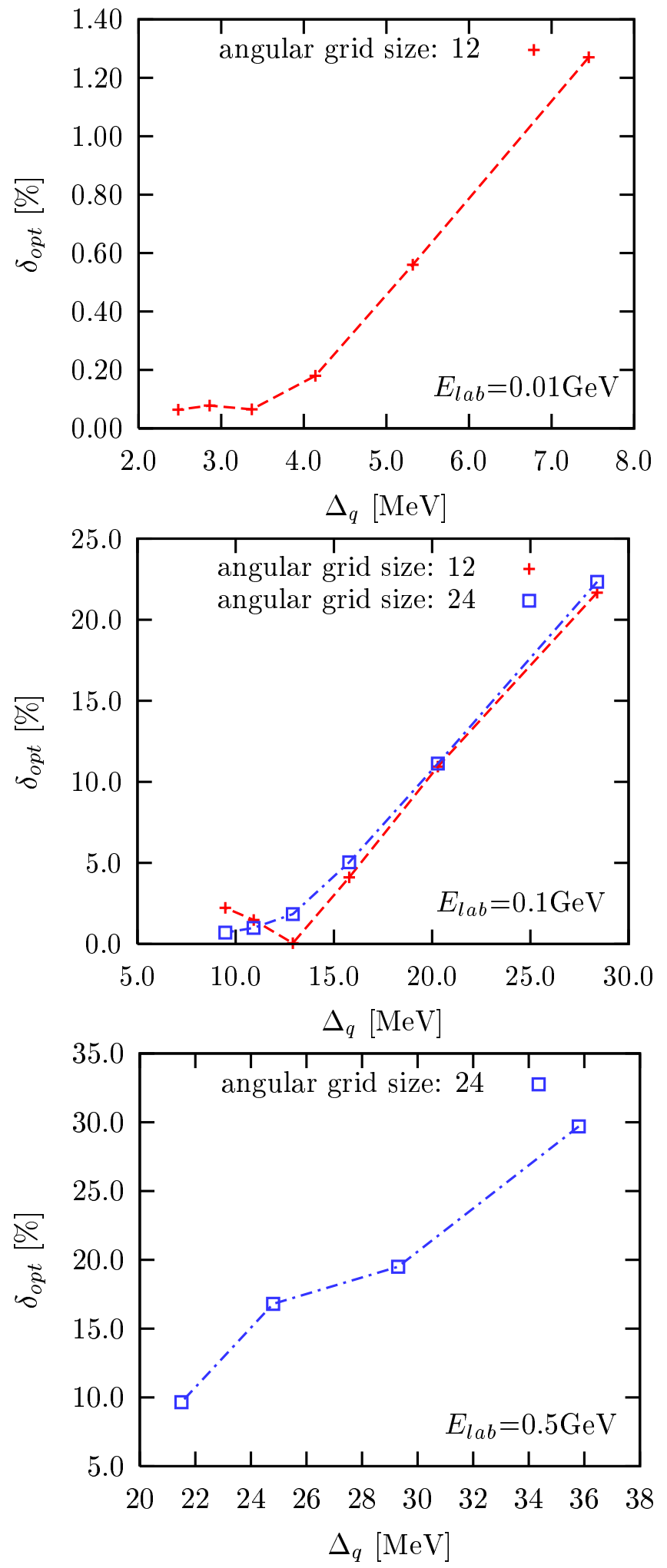

FIG. 3: The percent error in the optical theorem as function of the average distance $\Delta_{q}$ of the integration grid points $q^{\prime \prime}$ in the interval $\left(0, q_{m} a x\right)$ at the selected laboratory projectile energies $E_{l a b}=0.01 \mathrm{GeV}$ (top panel), $E_{l a b}=0.1 \mathrm{GeV}$ (middle panel), and $E_{l a b}=0.5 \mathrm{GeV}$ (bottom panel). 


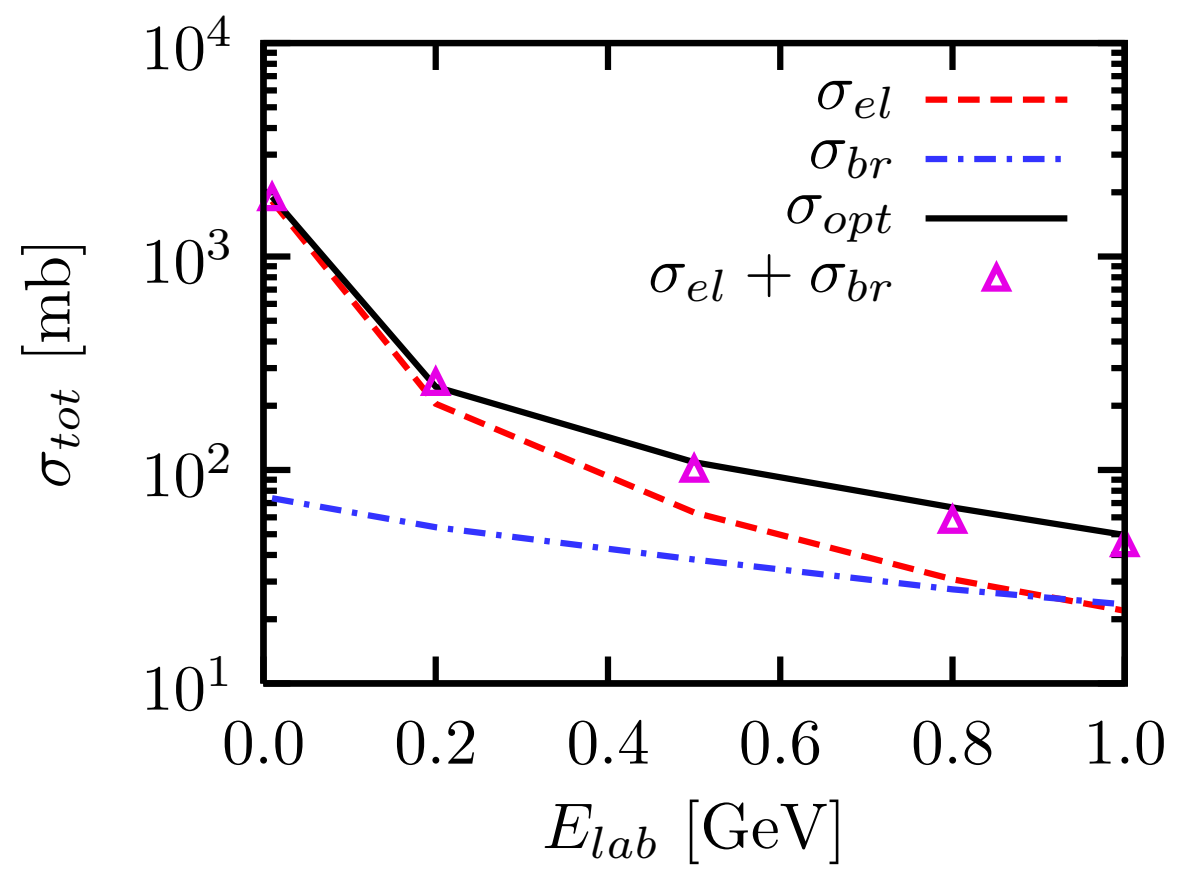

FIG. 4: The total elastic cross section $\sigma_{e l}$ (dashed line), the total break-up cross section $\sigma_{b r}$ (dash-dotted line) and the total cross section evaluated by the optical theorem $\sigma_{\text {opt }}$ (solid line) given as function of the projectile laboratory energy. At selected energies where the calculations have been carried out the sum of the calculated total elastic and break-up cross section, $\sigma_{t o t}=\sigma_{e l}+\sigma_{b r}$, is indicated by the open diamond. The open diamonds coincide with the solid line according to the optical theorem, Eq. 3.23 and the numerical values are given in Table II 

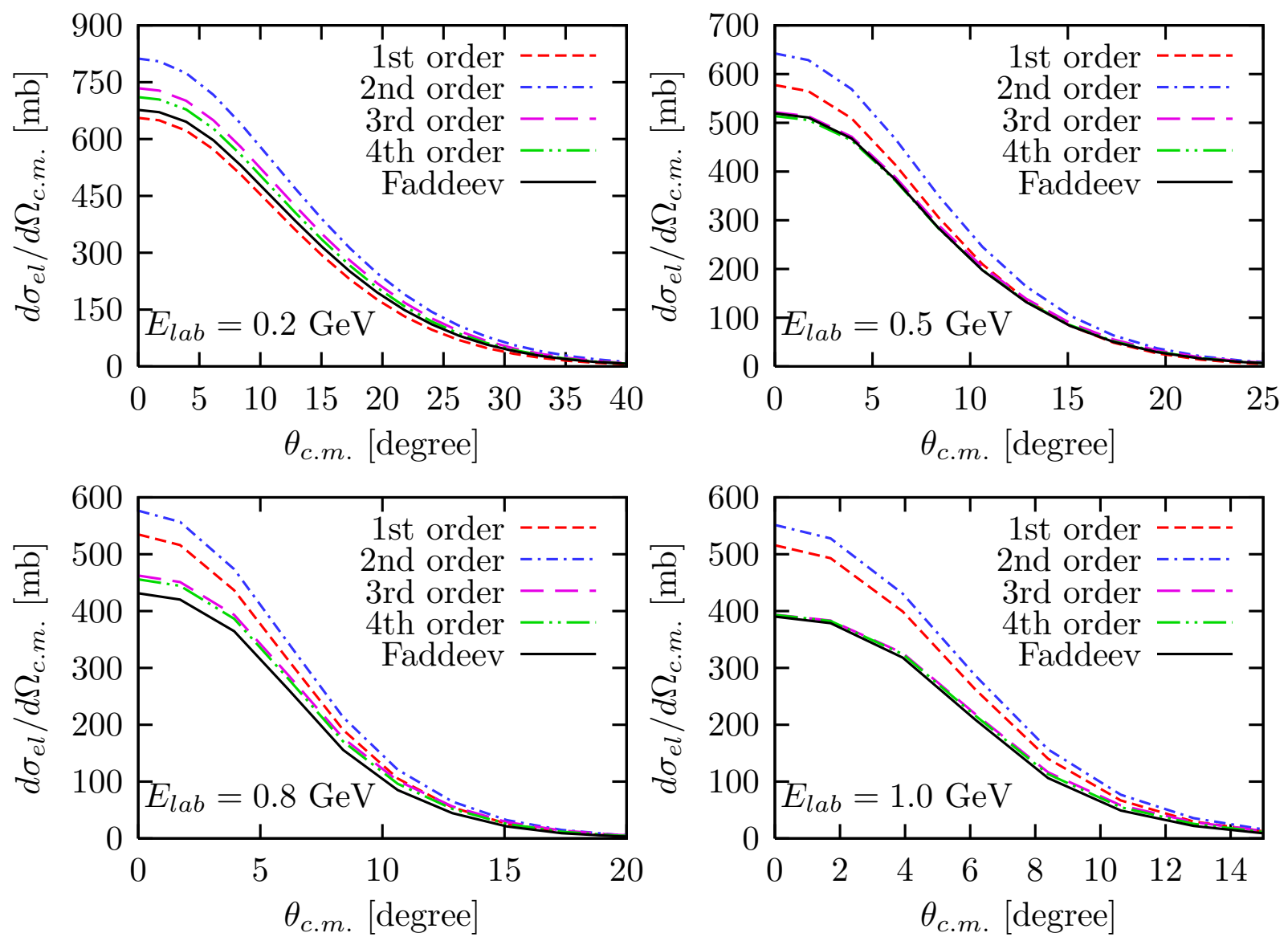

FIG. 5: The elastic differential cross section at $0.2 \mathrm{GeV}, 0.5 \mathrm{GeV}, 0.8 \mathrm{GeV}$, and $1.0 \mathrm{GeV}$ projectile energy as function of the laboratory scattering angle. In all cases the solid line represent the full solution of the Faddeev equation. The other lines represent the successive sum of different orders in the multiple scattering series, short-dashed the first order, dash-dot adds up to the 2 nd order, long-dashed to the 3rd order, and dash-dot-dot to the 4th order contribution. 

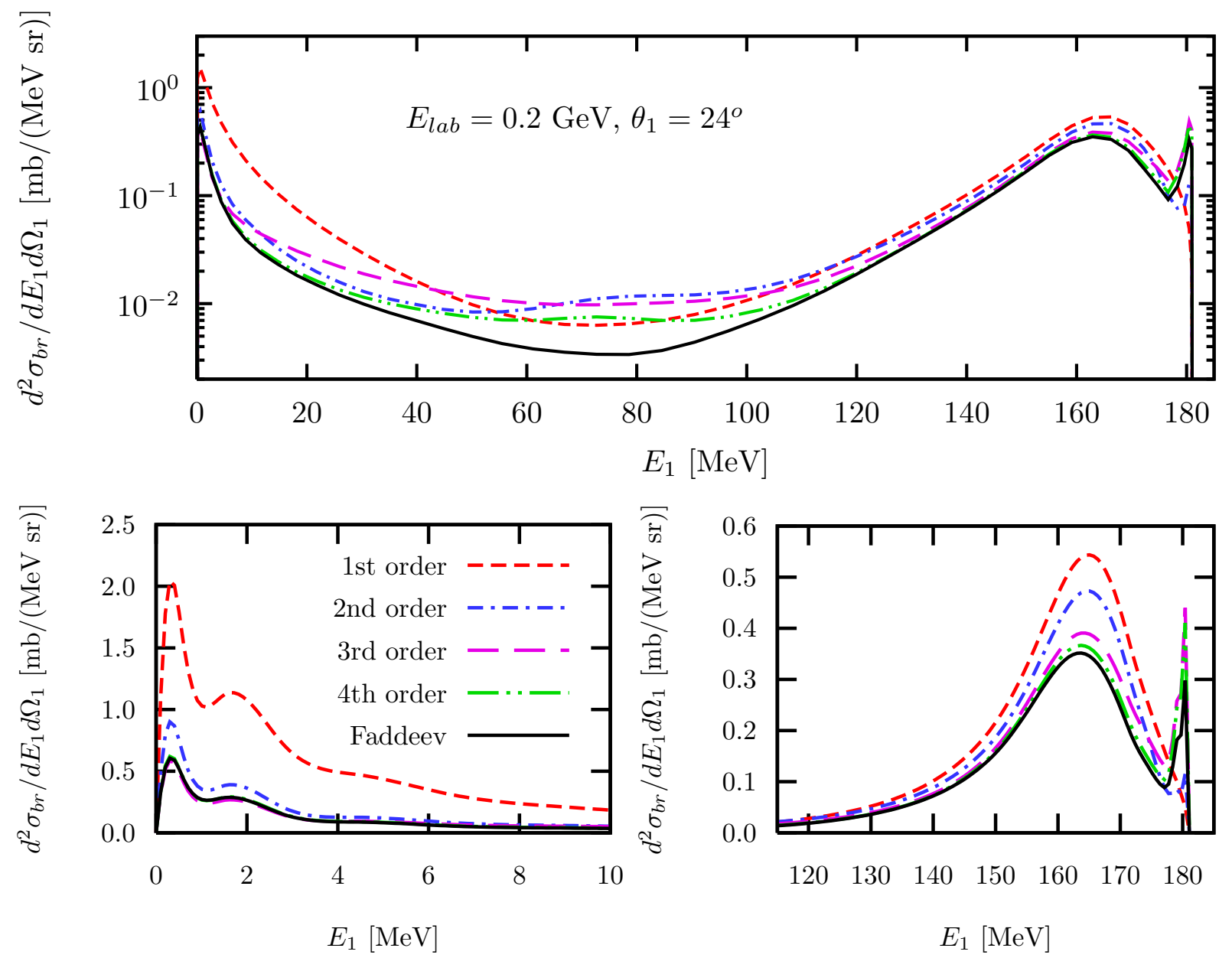

FIG. 6: The semi-exclusive cross section at $0.2 \mathrm{GeV}$ laboratory incident energy and at $24^{\circ}$ emission angle of the emitted particle. The upper panel displays the entire energy range of the emitted particle, whereas the two lower panels show only the low and high energies in a linear scale. The full solution of the Faddeev equation is given by the solid line in all panels. The contribution of the lowest orders of the multiple scattering series added up successively is given by the other curves as indicated in the legend of the lower left panel. 



FIG. 7: Same as Fig. [6 but for an angle of $39^{\circ}$ of the emitted particle. 

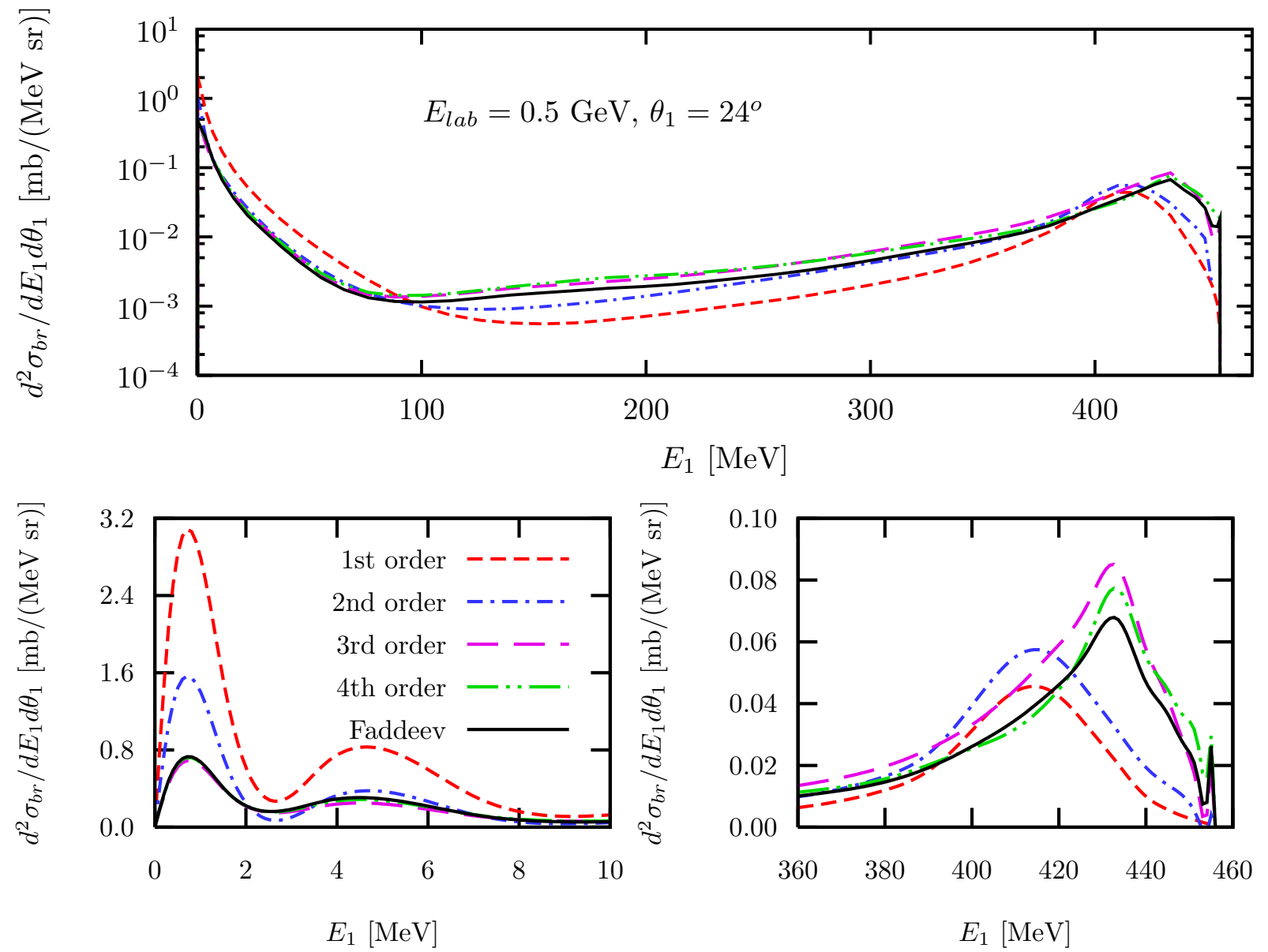

FIG. 8: The semi-exclusive cross section at $0.5 \mathrm{GeV}$ laboratory incident energy and at $24^{\circ}$ angle of the emitted particle. The upper panel displays the entire energy range of the emitted particle, whereas the two lower panels show only the low and high energies in a linear scale. The full solution of the Faddeev equation is given by the solid line in all panels. The contribution of the lowest orders of the multiple scattering series added up successively is given by the other curves as indicated in the legend of the lower left panel. 

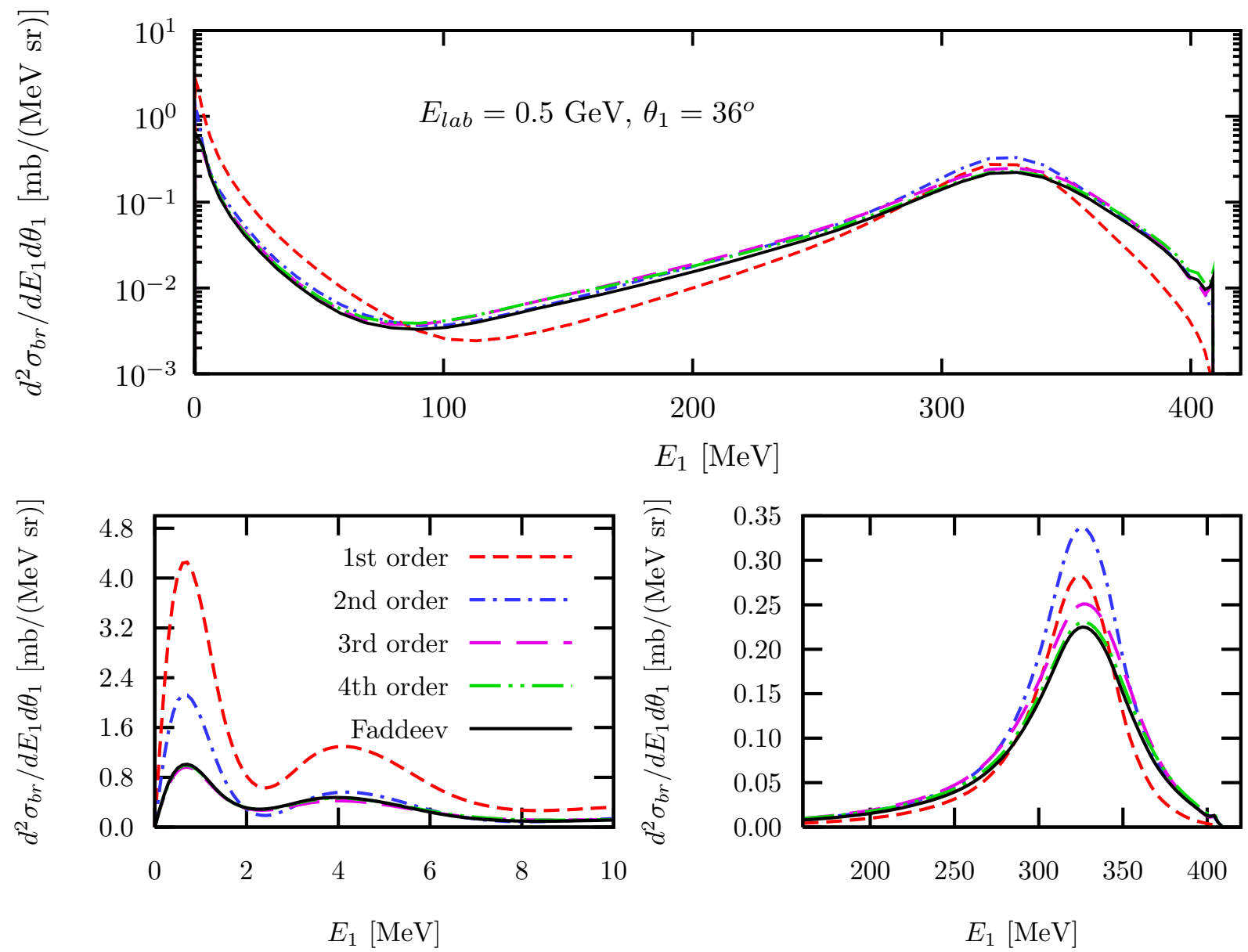

FIG. 9: Same as Fig. 8 but for an angle of $36^{\circ}$ of the emitted particle. 


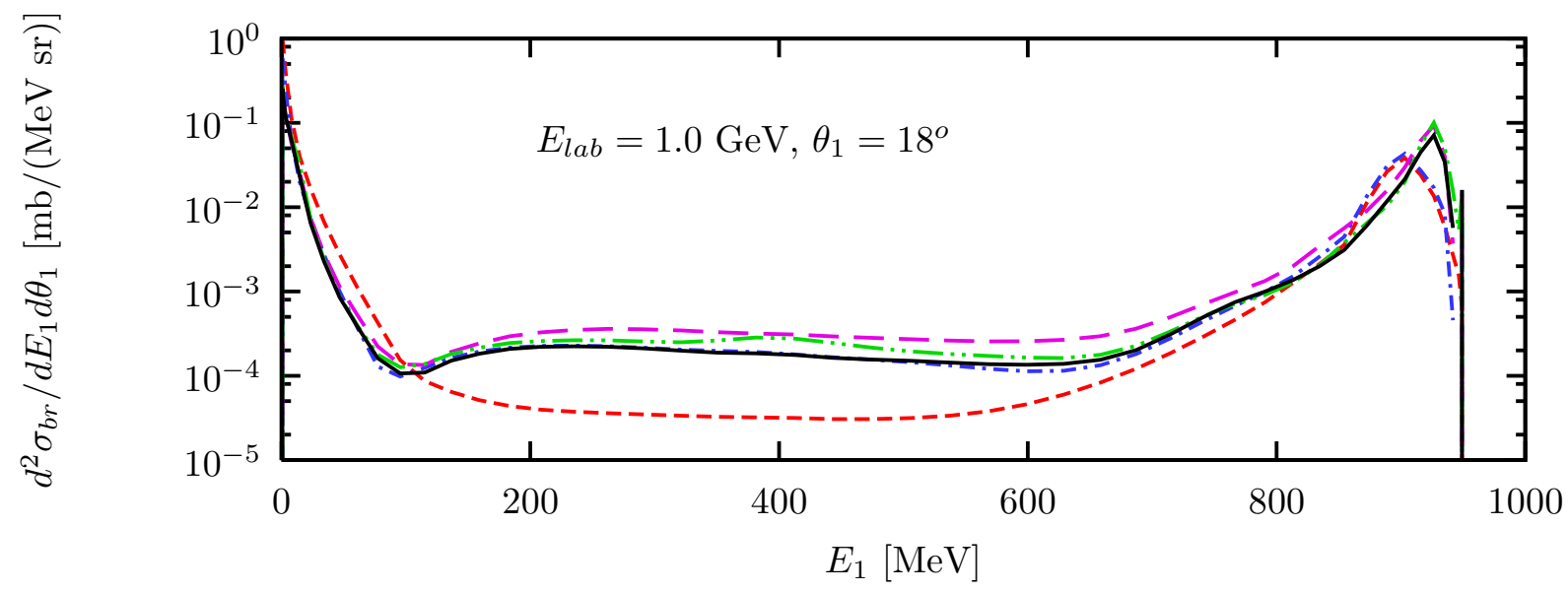

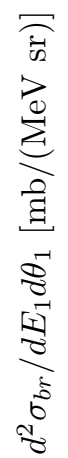
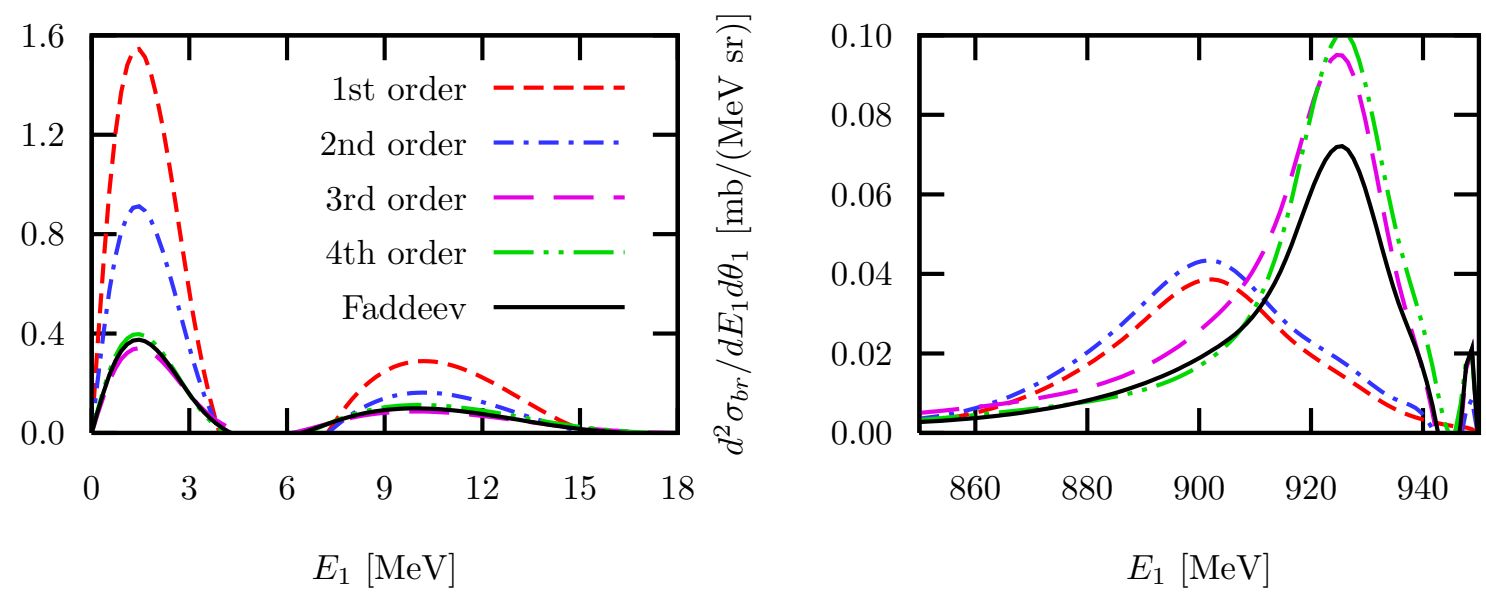

FIG. 10: The semi-exclusive cross section at $1 \mathrm{GeV}$ laboratory incident energy and at $18^{\circ}$ angle of the emitted particle. The upper panel displays the entire energy range of the emitted particle, whereas the two lower panels show only the low and high energies in a linear scale. The full solution of the Faddeev equation is given by the solid line in all panels. The contribution of the lowest orders of the multiple scattering series added up successively is given by the other curves as indicated in the legend of the lower left panel. 

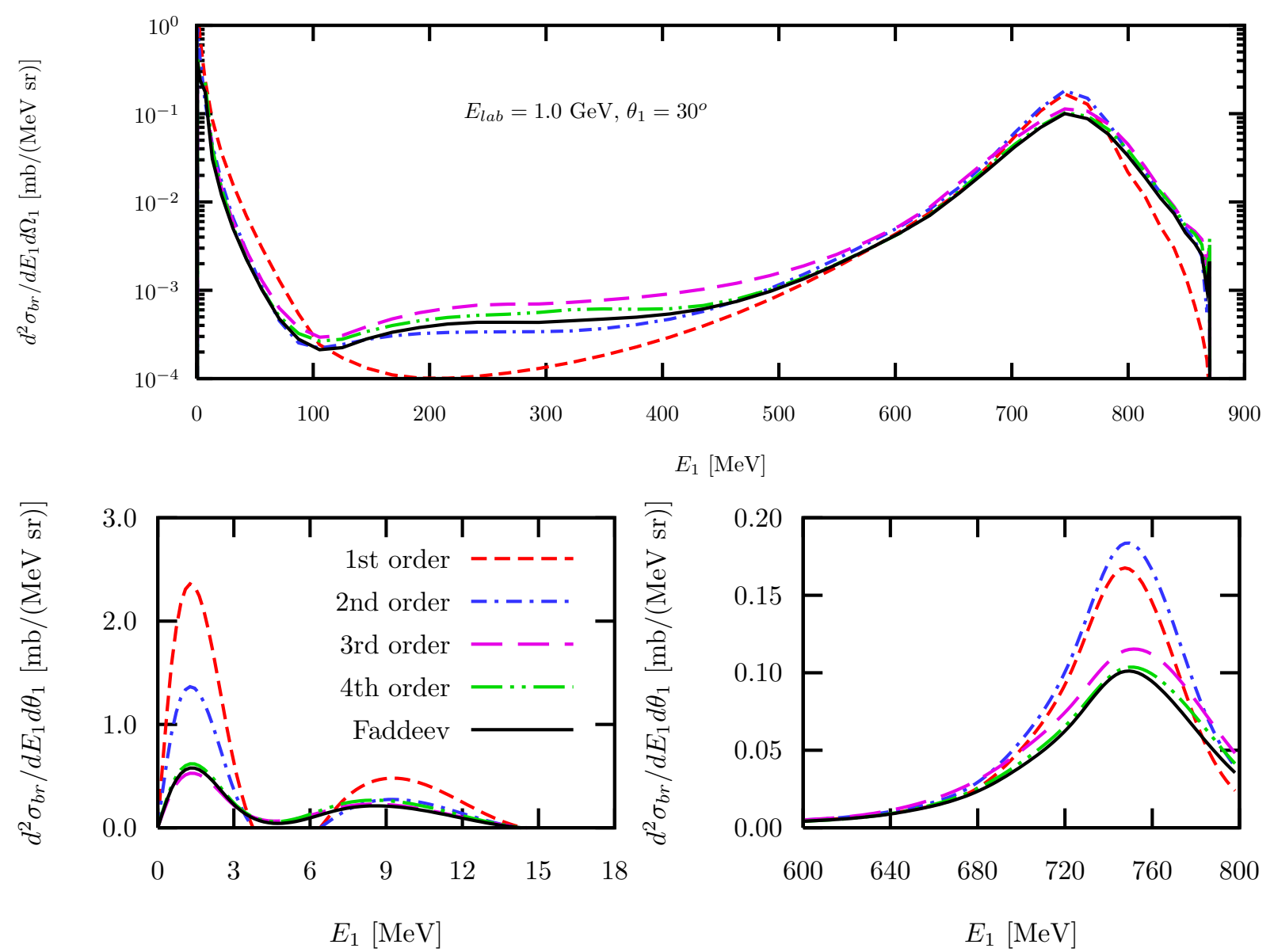

FIG. 11: Same as Fig. 10] but for an angle of $30^{\circ}$ of the emitted particle. 

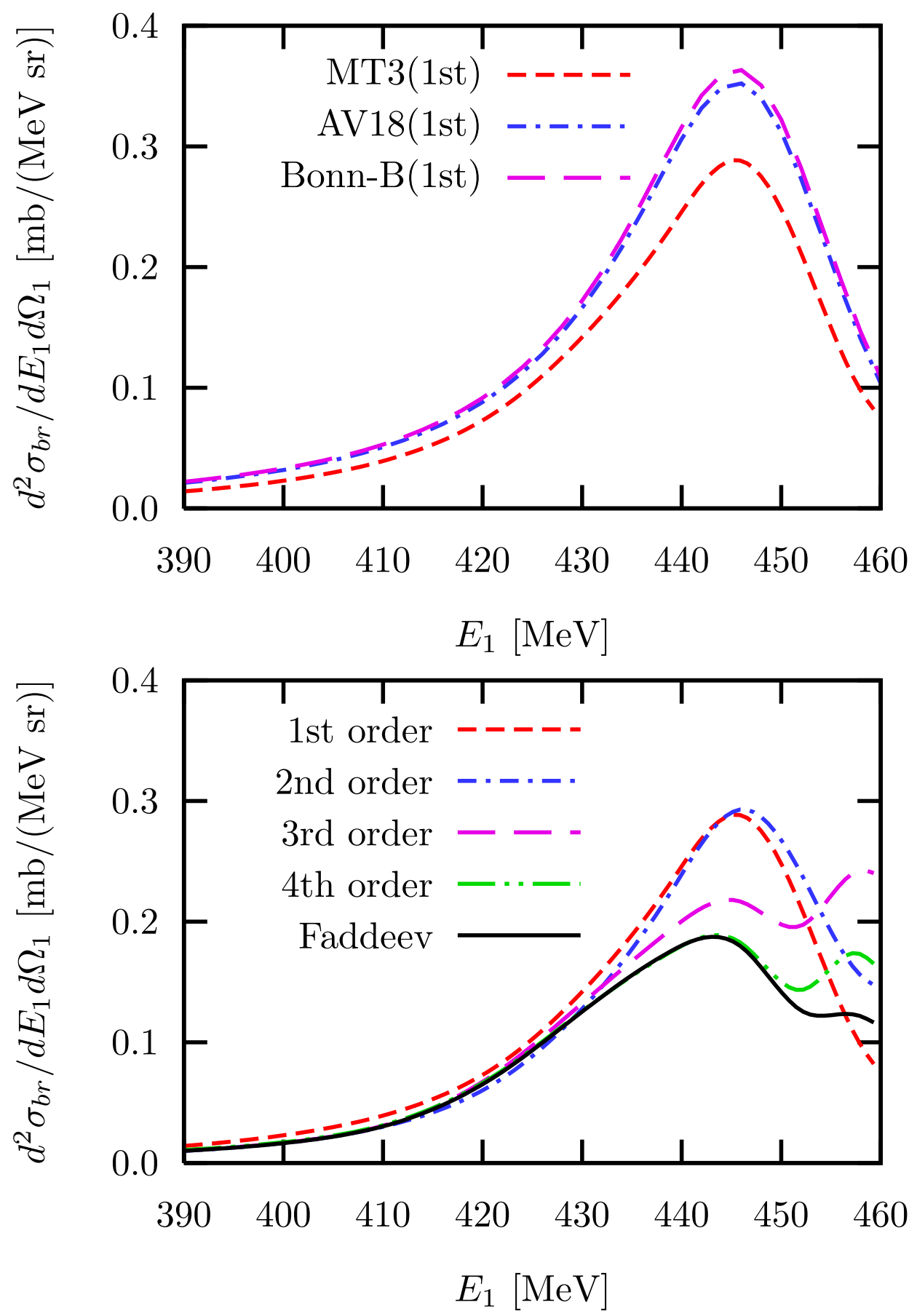

FIG. 12: The semi-exclusive cross section at $495 \mathrm{MeV}$ laboratory incident energy and at $18^{\circ}$ angle of the emitted particle. The upper panel displays the first order results obtained from the realistic potentials AV18 [27] (long dashed line) and Bonn-B [28] (dashed-dotted line) together with our calculation based on the scalar MT potential of Eq. [5.2 (dashed line). The lower panel displays again our first order calculation from the upper panel (dashed line), together with a successive addition of the next three rescattering terms. The exact solution of the Faddeev equation is given by the solid line. 


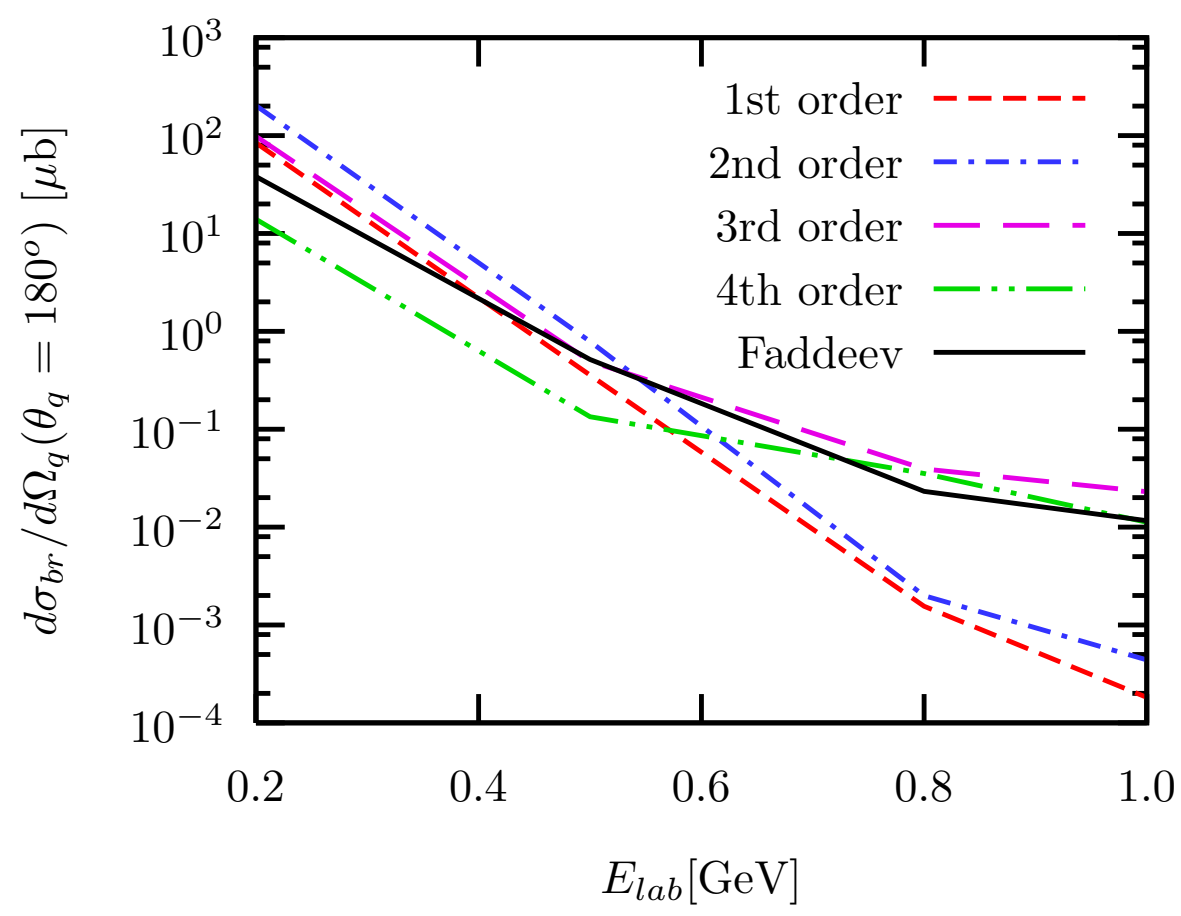

FIG. 13: The cross section (c.m.) for the semi-exclusive break-up reaction in which two particles emerge in forward direction with a relative energy between 0 and $3 \mathrm{MeV}$, and one particle is detected at backward angle as function of the projectile laboratory energy. The result of the full Faddeev calculation is given by the solid line and is compared with calculations based on the lowest orders of the multiple scattering series in $t$ added up successively as indicated in the legend.

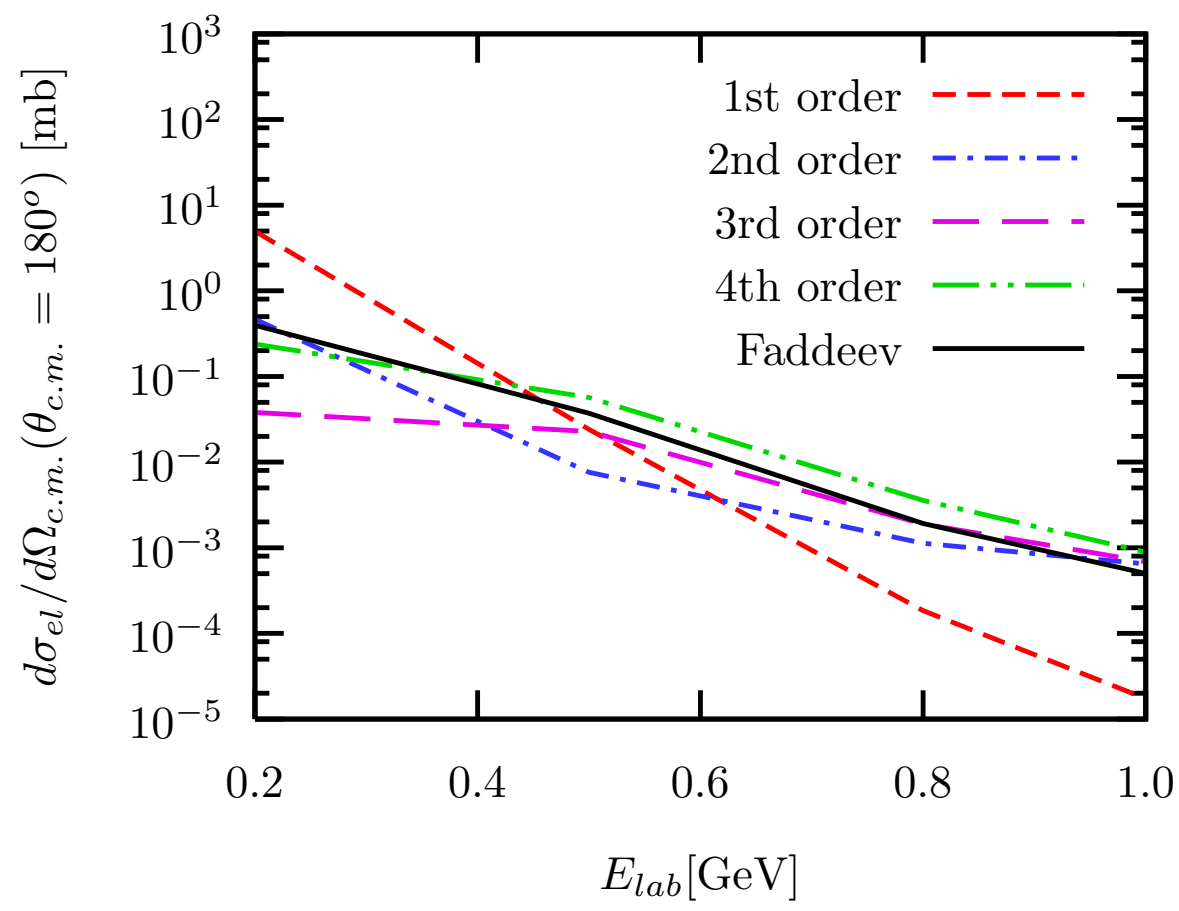

FIG. 14: The elastic cross section (c.m.) at backward angle as function of the projectile laboratory energy. The result of the full Faddeev calculation is given by the solid line and compared with calculations based on the lowest orders of the multiple scattering series in $t$ added up successively as indicated in the legend. 


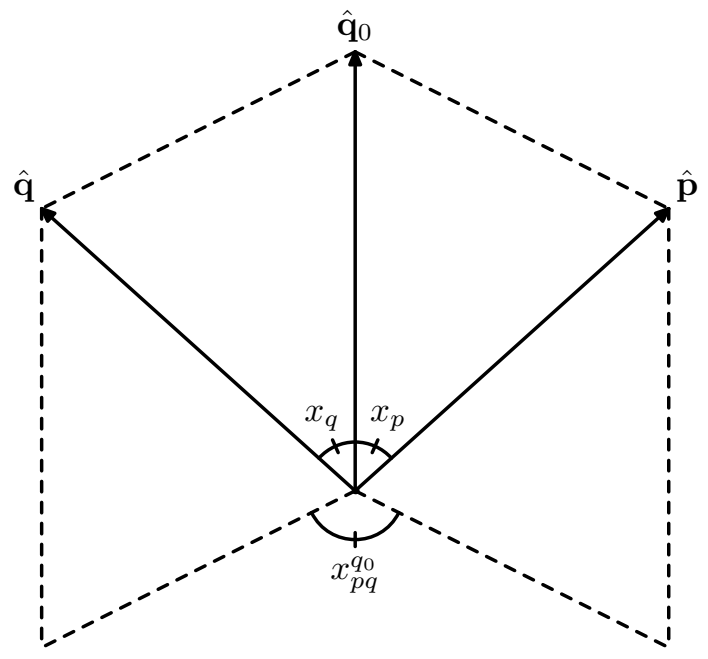

